\title{
Les Études aréales
}

Synthèse coordonnée par François-Joseph Ruggiu 


\section{Les Études aréales}

\section{François-Joseph Ruggiu (dir.)}

DOI : 10.4000/books. allianceathena. 457

Éditeur : Éditions A. Athéna

Lieu d'édition : Paris

Année d'édition : 2018

Date de mise en ligne : 19 janvier 2021

Collection : Collection ATHENA

EAN électronique : 9791093170121

\section{e OpenEdition}

\section{Books}

https://books.openedition.org

\section{Édition imprimée}

EAN (Édition imprimée) : 9791093170039

Nombre de pages : 84

\section{Référence électronique}

RUGGIU, François-Joseph (dir.). Les Études aréales. Nouvelle édition [en ligne]. Paris : Alliance Athena, 2018 (généré le 19 avril 2023). Disponible sur Internet : <http://books.openedition.org/allianceathena/ 457>. ISBN : 9791093170121 . DOI : https://doi.org/10.4000/books.allianceathena.457.

(c) Éditions A. Athéna, 2018

Licence OpenEdition Books 


\section{RÉSUMÉS}

À l'initiative du CNRS, les quatre Groupements d'intérêt scientifique dédiés aux études aréales (Études africaines en France ; Études asiatiques ; Institut des Amériques ; Moyen-Orient-Mondes musulmans) ont préparé, à partir de 2015, des livres blancs exposant les forces et les faiblesses du dispositif français de recherche en sciences humaines et sociales sur ces aires et en ont tiré plusieurs préconisations. Dans ce livre de synthèse, François-Joseph Ruggiu, directeur de l'INSHS, reprend les principales analyses de ces livres blancs et conclut sur les préconisations précitées. Les enseignements de cette synthèse concernent au premier chef les communautés scientifiques, les grands acteurs de l'enseignement supérieur et de la recherche et le ministère de l'Enseignement supérieur, de la Recherche et de l'Innovation. François-Joseph Ruggiu - directeur de l'Institut national des sciences humaines et sociales du CNRS. 


\section{Les Études aréales}

Synthèse coordonnée par

François-Joseph Ruggiu

Collection Athéna 
Collection Athéna, imaginée et dirigée par Françoise Thibault

Disputatio autour de la politique scientifique pensée comme un art d'organiser et de développer la recherche au bénéfice de la connaissance et des citoyens.

Dans la même collection

Des sciences dans la Science, ouvrage dirigé par Jacques Commaille \& Françoise Thibault

L'énergie des sciences sociales, ouvrage dirigé par Olivier Labussière \& Alain Nadaï

Illustration de couverture : «Continents » (C) Boise, 2017.

Responsable de publication : Simon Nataf

Charte graphique : Chloé Lepart

Composition graphique: Viviane du Guiny

Alliance thématique nationale des sciences humaines et sociales (Athéna), 2018.

Tous droits réservés.

ISBN : 979-10-93170-03-9

ISSN : 2425-2379

www.allianceathena.fr 


\section{Les Études aréales}

Synthèse coordonnée par

François-Joseph Ruggiu

Collection Athéna 



\section{Sommaire}

Introduction

Préconisations

21

L'état de la recherche : un constat différencié selon les aires et selon les disciplines.

Une question transversale :

la qualité de la formation

Les spécialistes d'études aréales:

des communautés aux besoins spécifiques.

Les recrutements

65 



\section{Introduction}

La capacité des chercheur.e.s ${ }^{1}$ à s'intéresser à des terrains d'enquête situés en dehors des frontières nationales, et à les étudier au plus haut niveau mondial, est un critère important de maturité et de qualité d'une communauté scientifique en sciences humaines et sociales (SHS). La France fait partie des nations dont les chercheur.e.s en SHS ont l'habitude de travailler sur des objets scientifiques inscrits dans des contextes situés partout dans le monde et de le faire en liaison étroite avec les communautés internationales spécialistes de ces objets. C'est un résultat qui s'appuie à la fois sur une longue tradition académique, sur une formation initiale qui laisse une

1. Les éléments développés ici synthétisent les apports des livres blancs produits par les quatre groupements d'intérêt scientifique (GIS) Institut des Amériques, Études africaines, Moyen-Orient et mondes musulmans et Asie, et consultables sur les sites web de ces GIS mentionnés plus haut. Nous remercions les directeurs de ces GIS, Jean-Michel Blanquer, Pierre Boilley, Catherine Mayeur-Jaouen ainsi que Sébastien Lechevalier et Jean-François Huchet, et toutes les personnes qui ont contribué à la rédaction et à la préparation de ces livres blancs, en particulier Marion Magnan, Véronique Lautier et Salomé Cheval, Cyrielle Michineau et Claire Le Poulennec. Nous remercions également l'Alliance thématique nationale des sciences humaines et sociales (Athéna) et sa déléguée générale Françoise Thibault de leur aide précieuse lors de la préparation de cet ouvrage et de l'accueillir dans ses collections. 
large place dans ses cursus à l'étude d'autres régions du monde que la France et l'Europe, sur l'élaboration dans des collectifs internationaux de théories, de méthodes, d'outils dans une dynamique de globalisation des recherches en SHS, et sur un investissement considérable de l'État. Les rares pays qui ont la capacité d'avoir des chercheur.e.s en sciences humaines et sociales capables de travailler sur des objets situés dans toutes les régions du monde y consacrent en effet des ressources importantes, comme, par exemple, aux États-Unis, où des fonds fédéraux ont été mobilisés en ce sens de manière très volontariste à partir des années 1990.

La France entretient de précieuses infrastructures de recherche qui permettent aux spécialistes d'un grand nombre de disciplines et de thématiques de travailler sur et à l'étranger. Àcôté des universités, nous pouvons mentionner ici les institutions spécifiquement tournées vers l'étude de grandes régions mondiales comme, par exemple, l'École française d'ExtrêmeOrient (EFEO) ou, sur des thématiques qui croisent les sciences humaines et sociales, l'Institut de recherche pour le développement (IRD). Nous pouvons également rappeler les dispositifs qui alimentent les recherches des personnels recrutés sur ces domaines au sein des organismes, comme le Centre national de la recherche scientifique (CNRS) ou l'Institut national d'études démographiques (INED), au sein des universités et des grandes écoles, en particulier l'École des hautes études en sciences sociales (EHESS), l'École pratique des hautes études (EPHE) ou l'Institut national des langues et civilisations orientales (Inalco), mais également 
les écoles françaises à l'étranger, les unités mixtes internationales du CNRS, ou les unités mixtes des instituts français de recherche à l'étranger (UMIFRE) opérées par le ministère de l'Europe et des Affaires étrangères et le CNRS. Enfin, les vastes ressources documentaires qui sont disponibles dans ou à partir des bibliothèques en France participent également de ce potentiel considérable.

Les chercheur.e.s français.es ou exerçant en France sont donc capables de faire progresser les connaissances mondiales soit sur des objets soit sur des terrains situés en Afrique, en Asie, sur le continent américain, ou encore dans le Pacifique. Ils les portent à un haut niveau car elles et ils associent la familiarité avec un terrain d'enquête ou d'étude, l'acquisition de la ou des langues de ce terrain, et la maîtrise d'une ou de plusieurs disciplines. L'articulation de ces trois éléments définit la nature même des études aréales telles qu'elles sont comprises dans ce texte. Cependant, cette situation n'est en rien un acquis définitif. Elle est, au contraire, fluctuante et semble avoir été remise en cause ces dernières années, à la fois pour des raisons internes à l'évolution de l'enseignement supérieur et de la recherche en France, et pour des raisons liées à la globalisation, qui transforme tant de secteurs depuis les années 1990. Les grandes et prestigieuses traditions d'étude du Moyen-Orient, des mondes musulmans, de l'Asie ou encore des Amériques, de l'Europe centrale et orientale, forgées en particulier à l'époque des Lumières, à celle de l'expansion coloniale, dont elles ont d'ailleurs été un des ressorts, et pendant les décennies qui ont suivi la Seconde Guerre mondiale et la décolonisation, 
restent vivaces et, pour l'essentiel, dynamiques. Mais elles doivent être sans cesse entretenues, développées et adaptées à l'évolution du monde académique contemporain. Et les points de fragilité de ces études doivent également et constamment être rappelés à l'attention des autorités académiques, aussi bien à l'échelle du ministère, qu’à celle des établissements, des communautés d'universités et d'établissements (Comue) et des communautés elles-mêmes.

Leur soutien est d'autant plus important qu'une des grandes forces des études aréales est de faire des chercheur.e.s qui y sont impliqué.e.s des interfaces entre les communautés scientifiques des pays qu'elles et ils étudient et les chercheur.e.s qui travaillent sur la France et l'Europe. Une autre spécificité des études aréales est leur communication étroite avec le tissu universitaire d'autres pays. Les circulations sont intenses, et la formation en France de nombreux.ses étranger. ère.s devenu.e.s par la suite chercheur.e.s ou enseignant.e.schercheur.e.s dans leurs pays est un vecteur fort de l'influence intellectuelle et universitaire française.

Pour ces raisons, les études aréales sont l'un des vecteurs les plus significatifs de l’internationalisation des SHS françaises. Cette dimension est d'autant plus précieuse que les spécialistes des différentes aires développent des compétences spécifiques et une expertise, appuyée sur leurs pratiques de chercheur.e.s, qui peuvent répondre de manière efficace aux besoins nouveaux du public et de l'État. L'internationalisation des SHS va donc de pair avec la question centrale du transfert 
des connaissances de la recherche publique vers la société civile et les pouvoirs publics.

Notre réflexion sur les études aréales croise une interrogation plus globale sur la recherche qui concerne les disciplines rares ${ }^{1}$. Dans certains domaines, siun.eprofesseur.e, un.edirecteur.rice de recherches ou un.e directeur.rice d'études parti.e en retraite n'est pas remplacé.e, un pan entier de recherche disparaît en France alors qu'il reste dynamique, par exemple en Allemagne ou dans les pays anglophones, ou se met en place et s'organise dans les pays émergents. La mutation d'un personnel administratif - comme d'un.e documentaliste - peut avoir strictement le même effet. Inversement, si un.e spécialiste de haut niveau formé.e en France ne trouve pas de poste dans notre pays, elle ou il pourra assez facilement trouver à s'employer temporairement ou définitivement à l'étranger, soit dans les universités des pays anglophones, soit dans les universités des pays émergents en matière de recherche, comme Singapour ou la Chine.

Le milieu de l'enseignement supérieur et de la recherche a réagi depuis plusieurs années à ces fragilités, en particulier en développant les groupements d'intérêt scientifique (GIS) tournés vers les études aréales : Études africaines en France ${ }^{2}$;

1. Voir le rapport Disciplines rares, remis à $\mathrm{M}^{\mathrm{me}}$ la secrétaire d'État chargée de l'Enseignement supérieur et de la Recherche le 16 décembre 2014. http://cache. media.enseignementsup-recherche.gouv.fr/file/Actus/62/1/rapport_final_mission_disciplines_rares_16-12-2014_404621.pdf

2. http://etudes-africaines.cnrs.fr/ 
Études asiatiques ${ }^{1}$; Moyen-Orient et mondes musulmans ${ }^{2}$, qui ont rejoint l'Institut des Amériques créé antérieurement ${ }^{3}$. Dotés d'un comité directeur, qui réunit les tutelles des unités de recherche affiliées au GIS, et d'un conseil scientifique, les GIS jouent un rôle important de coordination et d'optimisation de la recherche sur les grandes aires mondiales. Ils sont un lieu de discussion et de concertation entre les membres des communautés scientifiques concernées, à même de donner une meilleure visibilité à ces communautés et leurs travaux.

1. gis-reseau-asie.org

2. http://majlis-remomm.fr/

3. http://www.institutdesameriques.fr/fr 


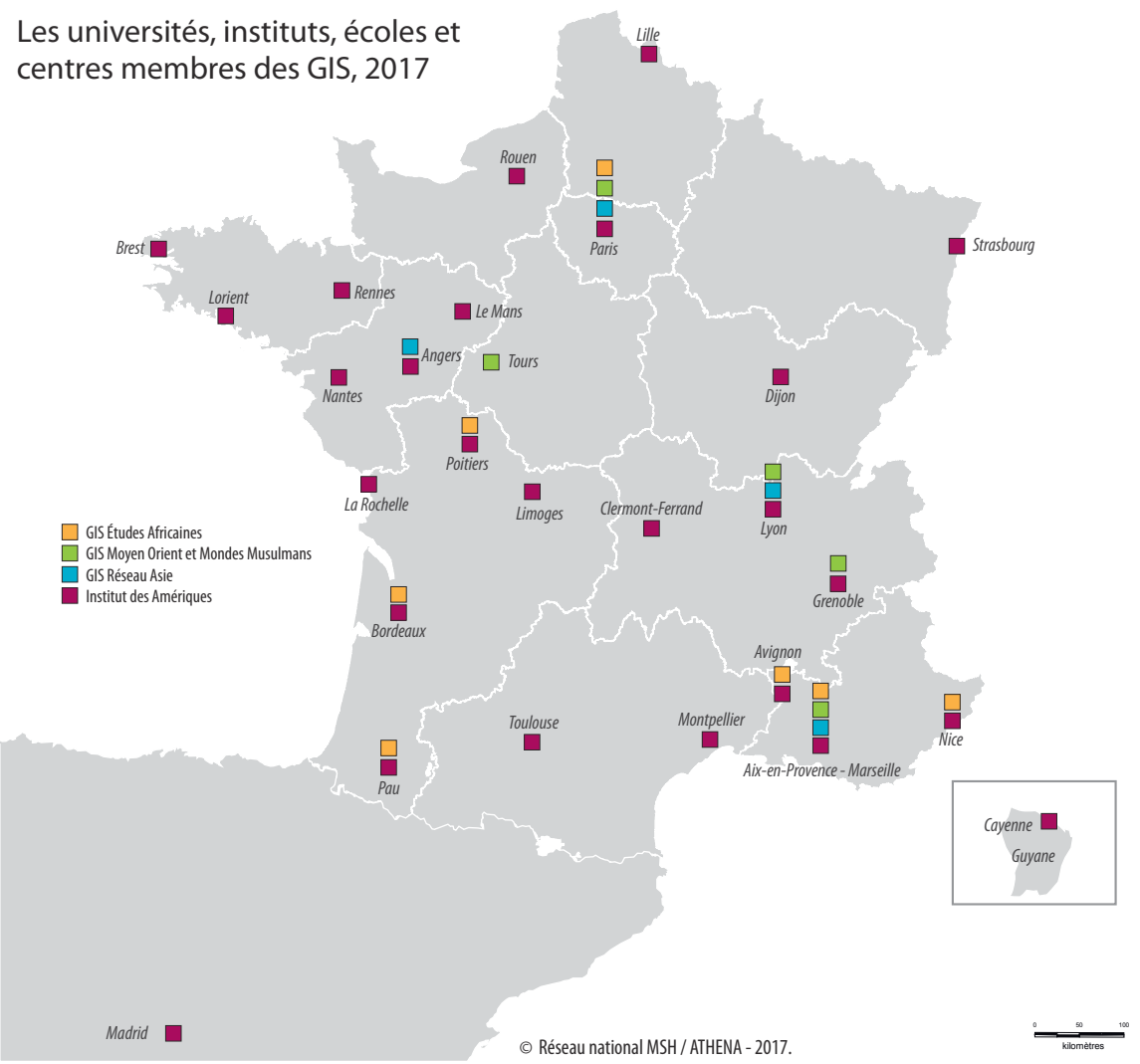




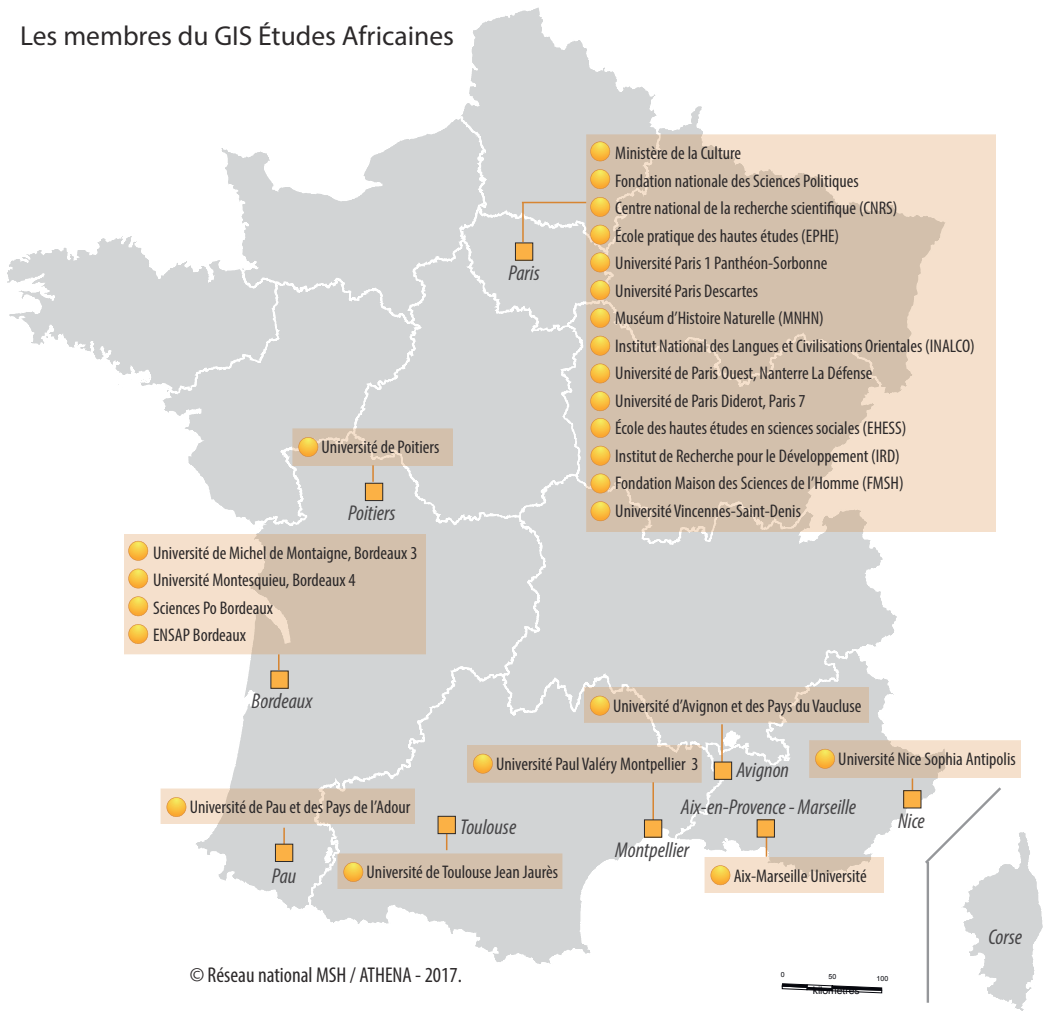




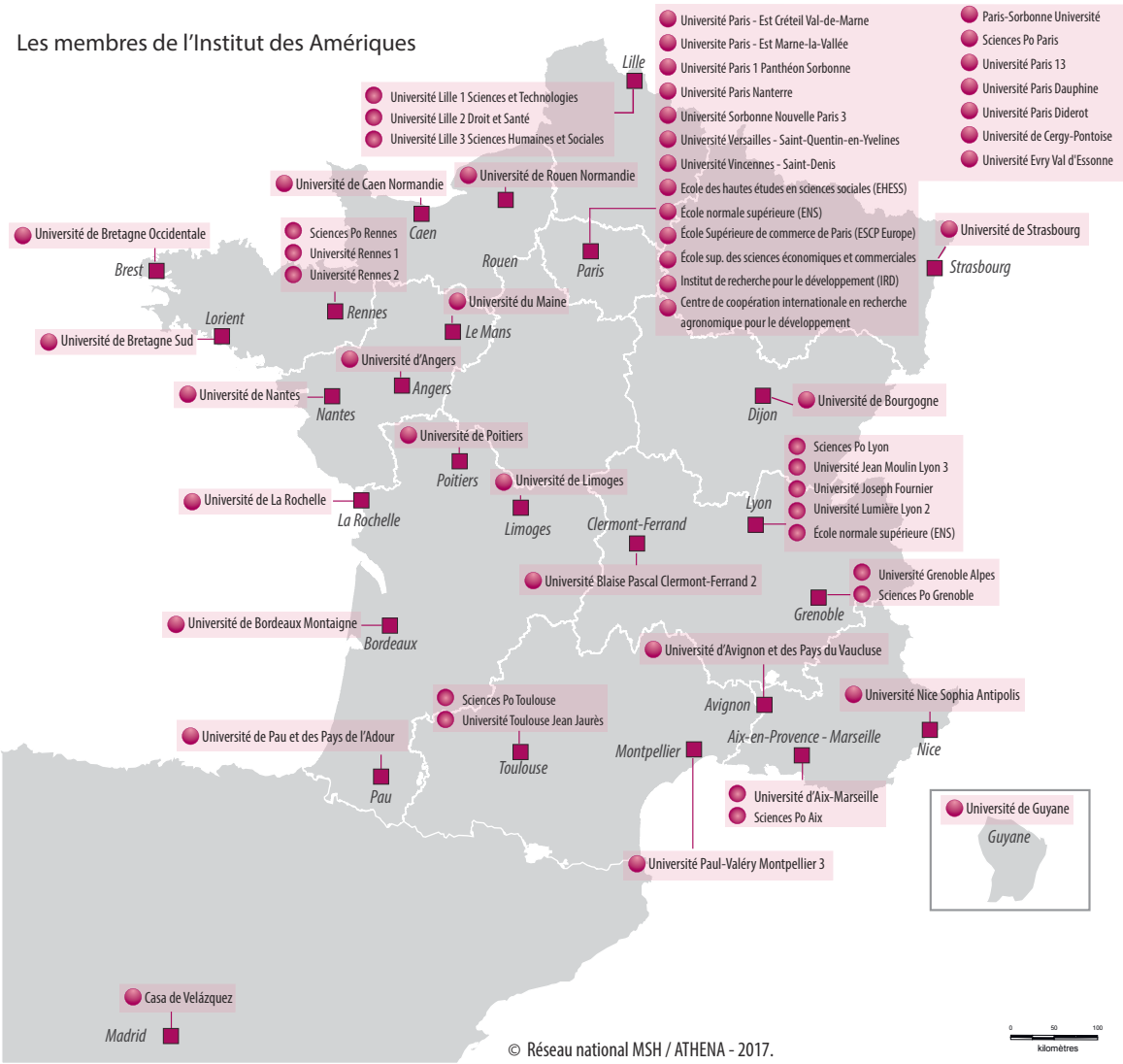




\section{Les membres du GIS Moyen Orient et Mondes Musulmans}

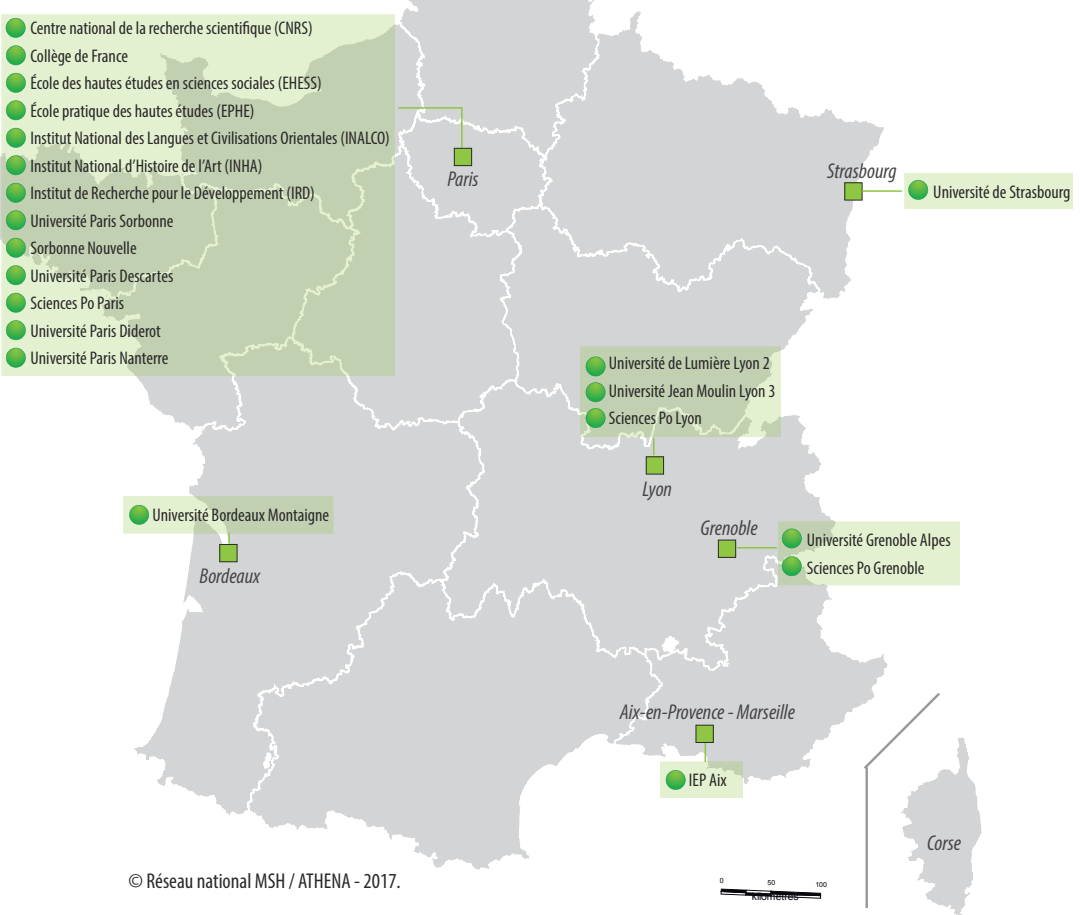




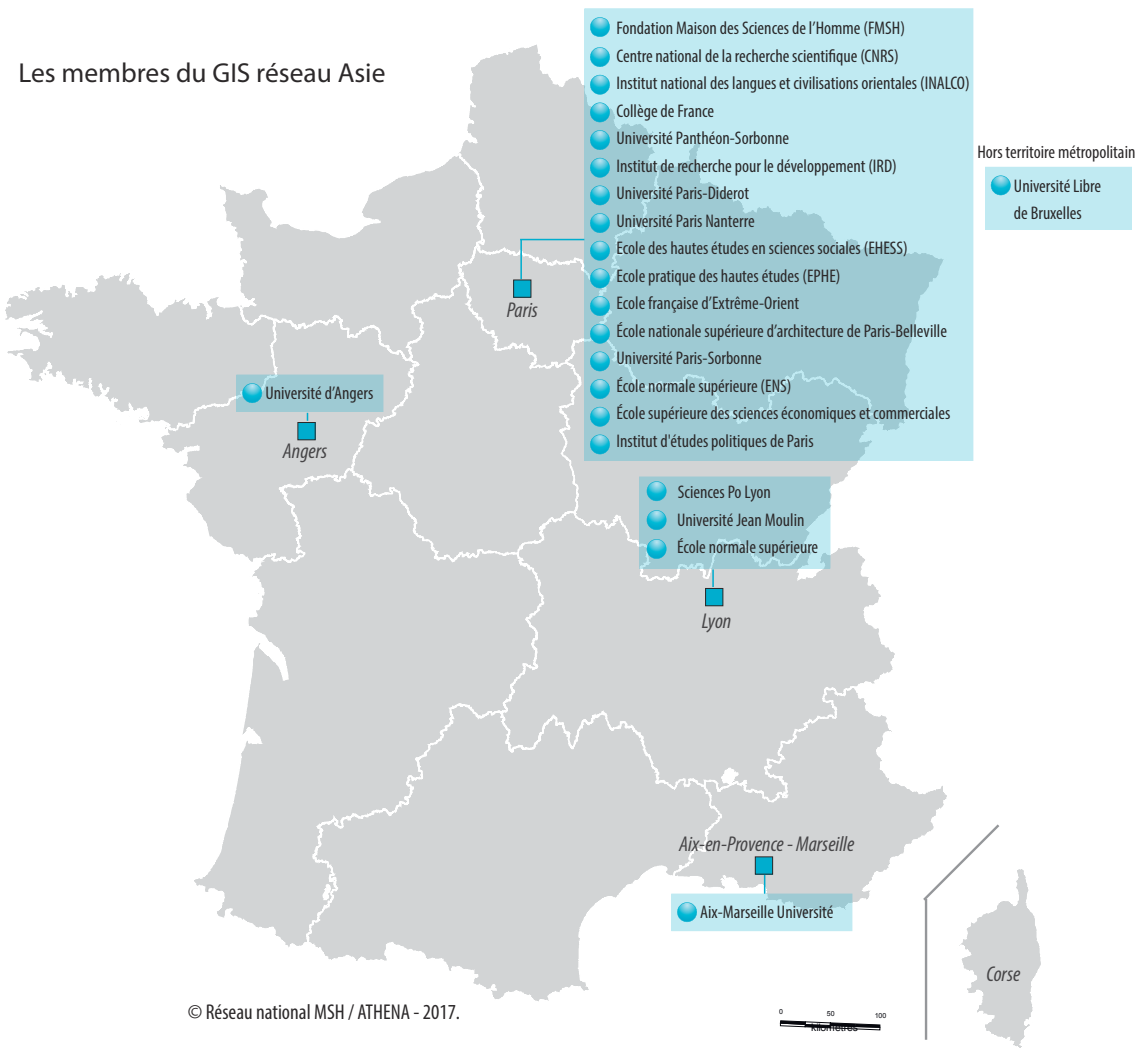


Les quatre GIS ont produit des livres blancs sur l'état des recherches sur les aires qu'ils couvrent et qui sont tous en ligne sur leurs sites web. Ils en ont tiré un certain nombre de préconisations concernant la préservation et le développement du potentiel de recherche de la France dans ces domaines. Ce potentiel est considérable mais il est confronté à deux défis : organiser la relève des générations dans un contexte de compétition internationale exacerbée ; et préserver et augmenter la puissance et la diversité thématiques, disciplinaires et aréales des recherches dans un contexte d'autonomie des établissements de recherche et de construction des politiques de sites. Sans prétendre les résumer, ce livre synthétise les principales analyses des livres blancs présentés par les GIS en études aréales et conclut sur un ensemble de préconisations qu'ils partagent. Pour un approfondissement et une analyse détaillée des situations à l'échelle régionale, nous renvoyons bien sûr les lecteurs aux quatre livres consultables en ligne sur les sites des différents GIS.

Les études aréales sont, parmi d'autres, un outil grâce auquel les communautés scientifiques françaises, quelles que soient les disciplines envisagées, entretiennent leur capacité de recherche sur les régions situées en dehors de l'Europe. Elles ne sont, naturellement, qu'un mode d'organisation de la recherche parmi d'autres. Elles ne doivent être considérées ni comme une proposition épistémologique à vocation hégémonique, ni comme une superstructure englobant toutes les formes de recherche sur les terrains lointains, et surtout pas comme une architecture contraignante qui cloisonnerait 
définitivement les aires. Les spécialistes mettent d'ailleurs depuis quelques années le dialogue entre les aires au cœur de leurs réflexions, en particulier entre spécialistes de l’Asie et de l'Afrique ou entre spécialistes des Amériques et de l'aire Asie-Pacifique. Elles et ils intègrent donc de plus en plus une intéressante dimension transaréale. D’ailleurs, au sein même des aires, des décloisonnements s'observent. Autrefois strictement séparé.e.s entre spécialistes de l'Amérique du Nord et spécialistes de l'Amérique latine, les chercheur.e.s américanistes s'organisent aujourd'hui de plus en plus en unités dédiées aux « Amériques », dans lesquelles la comparaison Nord/Sud est un axe fort des recherches. Enfin, les études aréales se sont révélées d'excellents vecteurs de réception, en France, des éléments les plus intéressants des grands courants mondiaux comme les études globales ou les études coloniales ou postcoloniales, menant à des formes de « désoccidentalisation » des SHS et de décloisonnement des recherches.

Les livres blancs des GIS en études aréales font le point sur l'organisation de la recherche sur les grandes régions du monde. Notons d'ailleurs qu'une partie de ces réflexions développées sur la spécificité de la recherche sur les aires africaines, américaines, asiatiques et pacifiques seraient valables également pour certaines parties de l'Europe, en particulier sur la Russie ou les Balkans. Les enseignements de ces livres blancs concernent donc au premier chef les communautés scientifiques et les grands acteurs de l'enseignement supérieur et de la recherche, du ministère de l'Éducation nationale, de l'Enseignement supérieur, de la Recherche et de l'Innovation 
(MESRI) aux établissements en passant par la Conférence des présidents d'université (CPU), l'Alliance thématique nationale des sciences humaines et sociales (Athéna), la Fondation Maison des sciences de l'homme (FMSH), ou les organismes comme le CNRS ou l'IRD. Mais, au-delà de ce premier cercle, ils doivent aussi intéresser l'ensemble des décideurs publics, en particulier au ministère de l'Europe et des Affaires étrangères (MEAE), partenaire de bien des actions que mentionnent les livres blancs, au ministère des Armées, au ministère de l'Intérieur, ou encore dans les assemblées parlementaires. 


\section{Préconisations}

Les livres blancs produits par les GIS consacrés aux études aréales ont mis en évidence le dynamisme des communautés scientifiques qui en relèvent. Il est servi par une meilleure structuration de la recherche au sein de laboratoires moins nombreux, mieux organisés, et bien soutenus par les pouvoirs publics.

Ils ont également relevé un changement récent du regard porté par les décideurs et par le public sur les chercheur.e.s en études aréales et en SHS en général. Les processus de mondialisation, les bouleversements globaux et interconnectés du monde, à l'image des transformations climatiques et de leurs conséquences sur les sociétés humaines ou de la grande crise économique de 2008, aussi bien que les évènements tragiques survenus depuis le début des années 2010 en France ${ }^{1}$, ont révélé le grand besoin de connaissances précises

1. Voir, à ce sujet, le rapport de l'Alliance Athéna, Recherches sur les radicalisations, les formes de violence qui en résultent et la manière dont les sociétés les préviennent et s'en protègent. État des lieux, propositions, actions, remis à M. le secrétaire d'État chargé de l'Enseignement supérieur et de la Recherche, mars 2016. 
et nouvelles sur l'Afrique, l'Asie, les Amériques ou encore le Pacifique.

S’ils nous livrent une cartographie précise des forces de la France dans ces domaines, ils relèvent également des lacunes et des points de fragilité que les autorités de l'enseignement supérieur et de la recherche doivent aborder ensemble à un niveau général aussi bien que local. Les préconisations faites ci-dessous s'inscrivent dans la dynamique du plan SHS présenté au cours de l'année 2017, en particulier les mesures 1 : accompagner l'évolution de la production des savoirs et leur diffusion ; 3 : faciliter l'accès aux financements par appels d'offres des SHS ; 7 : développer l'internationalisation des SHS et 10 : mettre les SHS au cour de la société1.

\section{Un développement plus intégré des formations}

La clé de l'avenir des études aréales est l'éveil des vocations chez les jeunes étudiant.e.s. Il convient donc de :

- Mettre l'accent dès la licence sur l'apprentissage des langues dans le cadre de cursus intégrés avec une formation disciplinaire (histoire, anthropologie, littérature comparée, etc.).

1. Voir Les SHS, un investissement pour l'avenir. 10 premières mesures en faveur des sciences humaines et sociales (SHS), communiqué de presse de M. le secrétaire d'État chargé de l'Enseignement supérieur et de la Recherche, 4 juillet 2016, http://cache.media.enseignementsup-recherche.gouv.fr/file/Actus/33/2/plan_ SHS_04-07-2016_604332.pdf 
- Encourager et mieux doter la création de doubles masters alliant compétences disciplinaires, compétences aréales et langues.

- Poursuivre cette formation auprès des doctorant.e.s et des chercheur.e.s en poste par des stages, ateliers ou summer schools, en apportant une attention particulière aux langues orientales.

- Créer davantage de contrats doctoraux et postdoctoraux, y compris pour des étudiant.e.s étranger.ère.s. Ces postdoctorats devraient être de longue durée (trois ans) et d'un haut niveau de rémunération. Les groupements d'intérêt scientifique consacrés aux études aréales devraient avoir la possibilité d'accorder des contrats doctoraux et postdoctoraux dans une perspective de coordination de la recherche sur le territoire national.

- Repenser l'insertion des chercheur.e.s des organismes dans les dispositifs d'enseignement. Les spécialistes d'aires ou de thématiques rares dans les organismes doivent être encouragé.e.s à enseigner, non seulement en donnant des séminaires, comme c'est le cas le cas à l'EHESS ou dans les Instituts d'études politiques (IEP), mais aussi en donnant des cours, y compris de niveau licence. 


\section{Un soutien ciblé à la recherche}

Un certain nombre d'opérations peuvent être prises en charge par les communautés elles-mêmes, en coordination avec les GIS en études aréales, comme, par exemple:

- Favoriser la mise en avant de la recherche par l'affichage plus systématique de prix de thèse ; par une incitation en direction des doctorant.e.s et docteur.e.s travaillant sur les aires à se porter candidats aux bourses générales (bourses du musée du Quai Branly, Fernand Braudel...) et aux bourses européennes (Marie Curie).

- Valoriser dans toutes les instances d'évaluation (Conseil national des universités (CNU), Comité national de la recherche scientifique (CoNRS) toutes les actions de transfert des communautés scientifiques mondiales vers la France. L'édition et la traduction de textes venus de langues autres qu'européennes doivent également être valorisées comme des tâches scientifiques nécessaires à la recherche.

- Utiliser tous les dispositifs d'accueil de chercheur.e.s étranger.ère.s, dont les durées sont variables : les onze chaires de l'Institut des Amériques (IDA) sur les Amériques, notamment à l'Institut des hautes études de l'Amérique latine (IHEAL), mais aussi dans diverses universités parisiennes, à Toulouse et à Rennes ; les accueils d'un an des Instituts d'études avancées français (IEA de Lyon, Nantes, Marseille, Paris) ; les accueils d’un mois réalisés par l'EHESS ou les 
universités ; les chaires du Collège d'études mondiales de la FMSH.

- Encourager la participation plus systématique des chercheur.e.s français.es à tous les congrès internationaux et intensifier leur organisation en France.

- Encourager la présence des chercheur.e.s français.es dans les revues internationales, et favoriser la publication de recherches élaborées en France dans ces revues.

D'autres actions doivent être menées avec le soutien du ministère de l'Enseignement supérieur et de la Recherche et de l'alliance Athéna, en particulier :

- Évaluer les apports des laboratoires d'excellence (Labex) en matière d'études aréales et penser la poursuite de certains d'entre eux.

- Soutenir les bibliothèques spécialisées dans les études aréales à la fois en personnels et en moyens, pour assurer le meilleur accès possible aux ressources documentaires, avec une attention particulière pour les ressources numériques.

- Assurer un suivi systématique de la situation de l'emploi dans les études aréales ainsi que des profils des recrutements effectués par les organismes, les grandes écoles et les universités. 
- Construire les structures de concertation qui permettront d'assurer le maintien de l'emploi et la diversité de sa répartition sur le territoire national.

\section{Une stratégie nationale de recrutement pour les études aréales}

L'enjeu que représentent les postes de chercheur.e.s et d'enseignant.e.s-chercheur.e.s est crucial dans un contexte qui, globalement, se rapproche de celui des disciplines rares. Les différentes branches des études aréales sont généralement portées par un petit nombre de personnes, ce qui rend les équilibres très fragiles.

Les viviers de recrutement pour le CNRS, les grandes écoles et les universités doivent être au minimum européens, voire internationaux et, dans tous les cas, ne pas se limiter à la France.

La politique des congés pour recherches et conversions thématiques (CRCT) attribués par les établissements comme les délégations attribuées par les organismes (CNRS, mais aussi INED ou IRD) doit être orientée de manière à favoriser les départs sur le terrain d'universitaires, en lien, lorsque cela est possible, avec les UMIFRE du CNRS et du ministère des Affaires étrangères. L'Institut universitaire de France (IUF), dont l’impact à long terme sur le développement de recherches de haut niveau est considérable, ne peut rester à l'écart de la mise en place de politiques scientifiques convergentes à l'échelle nationale en matière d'études aréales. 
Pour les recrutements disciplinaires dans l'enseignement supérieur, il convient aussi de désacraliser l'agrégation et le CAPES, des concours dont l'intérêt et la qualité ne sont évidemment pas en cause, pour mettre en évidence les savoirs linguistiques, la connaissance du terrain, les qualités proprement scientifiques, l'innovation ou encore un réel rayonnement international. La réussite à ces concours ne devrait pas être un critère décisif de recrutement des maîtres de conférences (MCF) dans les disciplines concernées.

Dans ces domaines hautement spécialisés, la question des recrutements se pose aussi pour les personnels des bibliothèques, ingénieurs, administratifs, techniques, sociaux et de santé (BIATSS). Il faut, en particulier, développer les postes de bibliothécaires et documentalistes spécialisé.e.s dans les études aréales, connaissant les langues orientales et capables d'être des interfaces entre les mondes étudiés, leur marché de l'édition scientifique, leurs bibliothèques, leurs ressources numériques, et les chercheur.e.s basé.e.s en France. Mais tous les postes sont concernés, à l'image des ingénieurs de la branche d'activité professionnelle (BAP) D ou des gestionnaires des unités.

\section{Le rôle des nouvelles institutions : vers une convergence des efforts?}

Il convient de réfléchir au rôle que pourraient jouer les Comue dans le développement des études aréales. 
Certaines d'entre elles, comme Aix-Marseille Université, Paris Sciences-et-Lettres, Université Sorbonne-Paris-Cité ou Hautes Écoles Sorbonne Arts et Métiers (HESAM) concentrent, en effet, une bonne partie du potentiel français dans ce domaine. Les trois dernières citées seront présentes sur le Campus Condorcet prévu à Aubervilliers en 2019.

Les études aréales doivent être un des axes privilégiés, en termes de formation et de recherche, de l'action des Comue et permettre des partenariats, des cohabilitations, des envois croisés de boursiers ou doctorant.e.s dans les pays étudiés.

Le rôle des GIS et de toutes les structures de coopération entre les chercheur.e.s (séminaires, associations de chercheur.e.s, etc.) doit être encouragé et leurs moyens doivent être renforcés. Le modèle du GIS Études asiatiques, qui peut s'appuyer sur l'unité propre de services (UPS) du CNRS « Réseau Asie » et dispose d'une importante capacité de gestion, de montage de projets et de soutien à l'édition, doit être développé.

Les interfaces entre les communautés scientifiques et la société doivent être multipliées. Le modèle de cette action peut être l'Institut d'études de l'Islam et des sociétés du monde musulman (IISMM) - unité mixte de services (UMS) 2000 CNRS/EHESS - fondé, en 1999, au sein de l'EHESS.

À l'international, il convient de favoriser à la fois la communication et la concertation entre les UMIFRE, peut-être grâce à un conseil scientifique commun, en encourageant des efforts 
convergents autour de projets partagés sans que l'ancrage local soit perdu. Les sites et les établissements de recherche et d'enseignement supérieur en France doivent être beaucoup plus solidement impliqués dans les projets des UMIFRE, notamment par des affectations ou des missions de longue durée d'enseignant.e.s-chercheur.e.s, et par la participation de ces sites à la définition des grands programmes scientifiques des UMIFRE. Dans ce cadre, cela peut induire que les sites fassent des choix parmi les aires géographiques et les UMIFRE, en termes d'investissement, pour éviter un éparpillement de leurs efforts. 



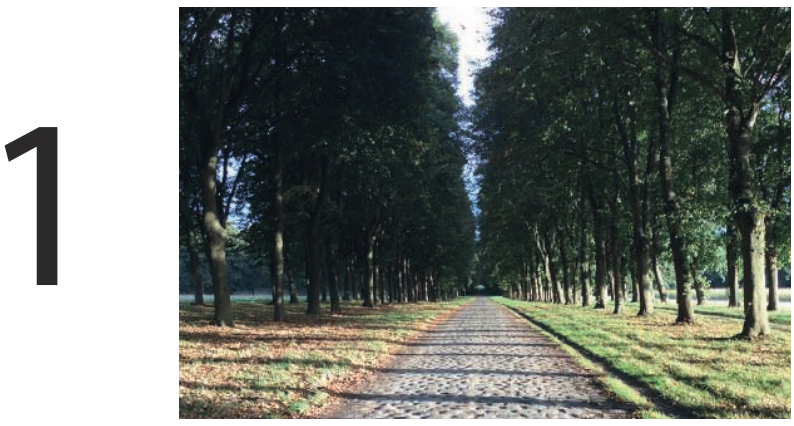

\section{L'état de la recherche : un constat différencié selon les aires et selon les disciplines}

\section{Analyse générale}

Dans les différents livres blancs, l’identification des secteurs qui se portent bien ou moins bien au sein de l'enseignement supérieur et de la recherche en France se fait par l'articulation de plusieurs critères : le nombre de chercheur.e.s qui s’y intéressent ; l'existence d'un nombre plus ou moins grand d'unités de recherche (unité mixte de recherche (UMR), équipe d'accueil (EA) sur le territoire national ; l'existence de formations dédiées, en particulier doctorales ; la présence d'un grand nombre de revues ou de collections consacrées à l'aire ou encore le nombre de thèses soutenues. 
Il est à noter que ces critères doivent être appréciés avec prudence. Par exemple, un grand nombre de revues sur une sous-aire peut davantage traduire la fragmentation des forces de recherches que l'abondance des travaux. À l'inverse, l'absence de revues sur un champ aréal donné peut amener les spécialistes de ce champ à publier soit dans des revues internationales soit dans des revues disciplinaires qui les mettent au contact d'autres aires et des recherches généralistes. L’idéal est bien sûr de croiser les différents modes de publication (livres, articles dans des revues de profils variés, chapitres d'ouvrage, éditions de textes, supports numériques, etc.) et de considérer les revues françaises et les revues étrangères.

Les livres blancs des différentes aires identifient en détail l'évolution des recherches dans les différentes disciplines et nous nous contenterons dans ce livre de souligner un ensemble de points forts et de points faibles communs aux différentes aires. Ces analyses doivent naturellement être lues à la lumière des spécificités de chaque communauté de chercheur.e.s. Les géographes ou les économistes semblent ainsi ne pas privilégier, dans la structuration de la recherche, l'approche aréale par rapport à l'approche disciplinaire ou thématique, à la différence, par exemple, des archéologues, des politistes, des anthropologues ou des historiens.

Une observation commune à l'ensemble des livres blancs est la capacité des communautés à se repenser depuis les années 1990. Comme le montrent l'évolution des unités de 
recherche, ainsi que leurs changements d'appellation, les visions traditionnelles des mondes africains, asiatiques, ou encore méditerranéens ont progressivement cédé la place à des analyses beaucoup plus nuancées, dynamiques et connectées aux problématiques développées sur les sociétés occidentales. Le passage d'« Amérique du Nord » et d' « Amérique latine » aux «Amériques » est très emblématique de ce processus, comme celui d' « Afrique blanche » et d'« Afrique noire » aux « Afriques ». Les dernières manifestations de l'essentialisme, du culturalisme et de l'exotisme ont définitivement disparu et les recherches sur les aires bénéficient en particulier du même haut degré de théorisation que les recherches menées dans les contextes français ou européens. Les communautés scientifiques se sont pleinement saisies des nouveaux objets, par exemple les mouvements politiques ultracontemporains, le genre, l'information et la communication, le vivant et la santé ou encore les problématiques à l'interface climat/ environnement/société.

À l'échelle des aires, il est aisé, à titre d'exemple, d'énumérer quelques points forts ou études en plein essor dans le domaine des études sur le Moyen-Orient et les mondes musulmans, comme les études turques, le droit musulman, l'histoire médiévale. Mais il y a aussi des lacunes ou faiblesses alarmantes par rapport à la concurrence mondiale : islamologie et domaines de l'érudition; sciences sociales de l'Iran ; histoire du Maghreb à partir de sources arabes, littérature classique en général, toutes langues orientales confondues, enfin histoire de l'art en pays d'islam. 
Or, il est essentiel ici de bien comprendre que, dans la logique des études aréales, il n'y a pas de dissociation possible entre les différents secteurs de la connaissance, par exemple entre les sciences politiques ultracontemporaines qui prendraient en charge l'analyse des mouvements sociaux du monde arabe, de la Chine communiste ou de l'Afrique de l'Ouest, et l'étude de la philologie, des littératures anciennes ou des traditions orales. Elles sont toutes également indispensables pour une compréhension globale des sociétés étudiées dans le passé et dans le présent.

\section{Un regard contrasté sur les effectifs}

Un des principaux enseignements des livres blancs est que les effectifs des communautés de chercheur.e.s que nous évoquons, même lorsqu'elles sont dynamiques et innovantes, peuvent être dans telle ou telle discipline relativement minces, et, si l'on croise zones et disciplines, deviennent parfois squelettiques. Ainsi, les sociologues spécialisé.e.s sur l'Afrique ne sont-elles et ne sont-ils pas plus d'une vingtaine, généralement dispersé.e.s dans plusieurs laboratoires pluridisciplinaires. Le nombre de chaires sur l'histoire de l'Amérique du Nord diminue depuis de nombreuses années et, sur l'histoire du monde arabe contemporain, on ne compte plus que six ou sept professeurs des universités en France, dont seulement un professeur d'histoire du Maghreb (à Paris 1). Enfin, à la rentrée de septembre 2016, il n'y avait plus aucun professeur d'université en islamologie, ce qui est inquiétant, même si quatre directeurs d'études maintiennent la tradition de 
cette discipline à l'EPHE. L'organisation disciplinaire du système universitaire peut aggraver cette fragilité résultant du petit nombre de chercheur.e.s et assécher des filières de recrutement de doctorant.e.s. Ainsi, l'Institut de recherches et d'études sur les mondes arabes et musulmans (IREMAM), pourtant l'un des laboratoires les plus importants sur l'histoire du monde arabe, n'est plus en mesure d'encadrer des thèses en histoire depuis le départ en retraite du seul titulaire de cette discipline.

Pour prendre un exemple régional dans une perspective pluridisciplinaire, alors que les récents accords internationaux vont vraisemblablement amorcer une montée en puissance des études sur l'Iran et que vivent dans notre pays de nombreuses personnes de nationalité ou d’origine iraniennes, il n’y a aujourd'hui en France aucun spécialiste habilité à diriger des recherches sur l'histoire moderne et contemporaine ou sur la géographie de l'Iran, les deux seuls professeurs ayant enseigné ces disciplines étant désormais émérites. Toutes disciplines confondues, il y a trois habilitations à diriger des recherches (HDR) seulement dans l'équipe Mondes iranien et indien (sur l'histoire de l'Iran ancien, histoire de l'Iran timouride, linguistique). La rentrée 2016 a heureusement vu deux recrutements au niveau professeur d'université (PU) à Paris 3 et Strasbourg. Si l'on ajoute une maître de conférences HDR, à Aix-enProvence, en linguistique persane, un sociologue directeur d'études à l'EHESS, un autre sociologue, professeur à Paris 7, nous avons fait le tour des spécialistes des sciences sociales de l'Iran contemporain, au niveau national, capables d'encadrer 
des recherches. C'est insuffisant et l'on pourrait dire la même chose des études turques, malgré le grand nombre d'habilitations actuellement soutenues sur la Turquie ou l'Asie centrale. La demande croissante des étudiants, de plus en plus nombreux à se spécialiser sur le monde turco-iranien, ne peut toutefois être encadrée. Dans un tel contexte, la France, malgré la qualité de son dispositif sur l'Iran (une UMR partiellement dédiée; l'Institut français de Recherche en Iran (IFRI) et sur la Turquie, peut difficilement envisager de rivaliser avec ses collègues états-uniens, britanniques ou allemands.

La mesure globale des recherches concernant l'Asie, dont les États couvrent près d'un tiers des terres émergées et abritent $60 \%$ de la population mondiale, est également riche d'enseignements. Les forces de la recherche publique française sur ces pays, toutes disciplines de sciences humaines et sociales confondues, sont concentrées dans 46 laboratoires, qui comptent, en tout et pour tout, 650 chercheur.e.s statutaires environ, auxquel. le.s s'ajoutent environ 730 doctorant.e.s et postdoctorant.e.s. Le nombre des personnels de soutien et de support à la recherche dans ces laboratoires ne dépasse pas la centaine.

Si nous descendons à une échelle plus fine, en regardant la situation des différents pays de l'Asie à travers les membres du Réseau Asie, animé par le GIS Études asiatiques, nous observons que la recherche française est concentrée principalement sur trois pays : la Chine, le Japon et l'Inde, qui représentent respectivement $21,5 \%, 13,5$ et 11,5\% du total des chercheur.e.s sur l'Asie. Ce sont les seuls pays réunissant 
plus d'une centaine de spécialistes. Pour d'autres pourtant particulièrement importants dans la géopolitique mondiale, comme les Corées, l’Indonésie ou la Thaïlande, le nombre de spécialistes en SHS en France oscille entre trente et quarante seulement, même si, ponctuellement, d'autres chercheur.e.s peuvent s’y intéresser sous un angle thématique.

Pour certains pays, comme le Pakistan, particulièrement difficile d'accès ces dernières années, mais aussi le Bangladesh, le Sri Lanka, le Laos, les Philippines, la Birmanie ou encore Taïwan, la couverture est très faible. On note également le peu d'intérêt pour les rapports entre l'Asie et l'Océanie, pourtant en évolution rapide, en raison de la présence chinoise notamment, et qui devraient intéresser la France, compte tenu de sa forte présence dans cette partie du monde.

Autre exemple, le nombre de chercheur.e.s intéressé.e.s par les Amériques semble assez élevé en apparence : 879 personnes. Ce chiffre est cependant en retrait depuis le recensement de 2009. Par ailleurs, on note, comme pour d'autres aires, un fort vieillissement qui pèse sur l'avenir. Enfin, si certains pays ou spécialités sont assez bien lotis, comme les études de management et gestion sur les États-Unis, d'autres le sont beaucoup moins. Dans certains cas, la situation est surprenante. Ainsi, un certain antiaméricanisme, fort jusqu'aux années 1990, et peut-être une certaine crainte de confronter les universitaires américains sur leur terrain ont eu pour conséquence le faible développement de l'étude des ÉtatsUnis dans certaines disciplines. 
On pourrait enfin citer le petit nombre de professeurs d'université sur l'Afrique. À l'université Paris 1, où cette spécialité est bien présente, si l'on prend par exemple le cas de l'histoire, un professeur est présent pour l'histoire ancienne, de l'Antiquité à l'époque moderne, et un autre doit encadrer les thèses concernant l'ensemble du continent à l'exclusion de l'Afrique du Nord, du XVII ${ }^{\mathrm{e}}$ au XXI ${ }^{\mathrm{e}}$ siècle. La spécialisation par ensembles régionaux, et a fortiori par pays, reste donc inenvisageable.

Les analyses développées ici ne sont, bien sûr, en rien des jugements de valeur sur la qualité intrinsèque des recherches scientifiques menées par tels ou tels communauté, discipline et champ de recherches. Il n'est pas rare, par ailleurs, qu'un secteur ou une discipline identifiés dans les livres blancs comme en crise ou en déclin à l'échelle nationale ou sur certains sites puisse se prévaloir de personnalités tout à fait reconnues et actives à l'échelle internationale.

\section{3. Études aréales et politique de site}

À ce propos, la répartition géographique des forces de recherche sur le territoire national témoigne, depuis plusieurs années, d'un fort tropisme parisien, encore inégal selon les disciplines mais qui ne cesse de se renforcer. Notons, par exemple, que près de la moitié des thèses soutenues annuellement en France sur l'Afrique (44\%) le sont à Paris. Les deux tiers des effectifs des laboratoires travaillant exclusivement ou partiellement sur l'Asie sont également situés à Paris ou en région parisienne. Les raisons de cette concentration sont 
connues : proximité des centres de ressources documentaires ; facilité des connexions avec le monde et les régions étudiées, en particulier pour les transports ; concentration des établissements de recherche et d'enseignement supérieur, et des séminaires de recherche tournés vers les études aréales, au premier rang desquels l'EHESS, l'EPHE, l'Inalco ou encore les universités de Paris 1 Panthéon-Sorbonne, ParisSorbonne, Paris Diderot-Paris 7, Paris 8 ou Paris-Nanterre. Il est vrai que les plus petits sites en région ont des difficultés à atteindre la masse critique de chercheur.e.s qui leur permettrait de peser sur les dispositifs d'enseignement et sur les profils de recrutement des MCF et professeurs.

Parmi les pôles régionaux qui continuent à abriter une recherche structurée en études aréales, Aix-en-Provence est servi par la Maison méditerranéenne des sciences de l'homme (MMSH) et par le choix scientifique fort qu'il a fait vers l'étude des sociétés et des populations de la Méditerranée (avec, en particulier, le Labexmed). Le site abrite également l'UMR Credo qui est la seule unité entièrement tournée vers le Pacifique (UMR CNRS, EHESS, Aix-Marseille Université (AMU). Le site de Lyon conserve aussi une place dans ce domaine, en particulier grâce à l'UMR Institut d'Asie orientale et à d'autres unités sur l'Asie, ainsi qu'aux unités d'archéologie et d'histoire consacrées à l'Orient, autour de la fédération de recherche Maison de l'Orient et de la Méditerranée $(\mathrm{MOM})$. Le site bordelais a gardé une réelle pertinence sur l'Afrique, grâce à une tradition ancienne (la Maison des Suds) et la présence de l'UMR Les Afriques dans le monde. 
Le site de Toulouse conserve une tradition de recherche sur les Amériques avec l'Institut d'études pluridisciplinaires des Amériques de Toulouse et la Maison universitaire francomexicaine, comme c'est le cas du site de Rennes avec l'Institut des Amériques-Rennes. Les autres sites en région ont parfois gardé soit une EA tournée vers une aire spécifique, soit des thématiques de recherche de niche, ou bien n'ont plus qu’une activité dispersée, liée au rayonnement d'un ou de plusieurs chercheur.e.s ou enseignant.e.s-chercheur.e.s de premier plan.

Pour reprendre l'indicateur des soutenances de thèses sur les mondes africains, les sites de Grenoble, Lille, Nice, Montpellier, Toulouse et Strasbourg ne représentent qu'entre 2 et $4 \%$ de l'ensemble. Pour l'Asie, la situation semble cependant plus contrastée. Si douze établissements parisiens (Paris 7, Paris 1, EHESS, Paris IV, Paris 3, Paris X, Paris 5, Paris 8, l'Inalco, Paris 2, EPHE et Sciences Po Paris) concentrent à eux seuls $61 \%$ des thèses soutenues sur l'Asie entre 1970 et 2013, cette polarisation semble avoir régressé depuis 2000 puisqu'ils ne représentent plus que $52 \%$ des thèses soutenues sur l'Asie entre 2000 et 2013 alors que certains pôles universitaires comme Aix-Marseille, Nice, Bordeaux, Lyon, Rouen, Nantes et Montpellier ont connu une forte progression durant la dernière période étudiée.

Dans l'ensemble, les Comue créées dans le sillage de la loi de 2013 sur l'enseignement supérieur et la recherche semblent avoir peu envisagé de placer explicitement une aire mondiale au coeur de leur programme scientifique. Il y a cependant 
d'importantes exceptions : AMU, qui a mis la Méditerranée au centre de sa stratégie générale de recherche ; HESAM, qui a présenté une thématique sur «Afrique(s) en devenir » parmi les grands axes du projet d'Initiatives-Science-Innovation-Territoires-Économie (ISITE) présenté dans le cadre du deuxième programme d'investissements d'avenir (PIA 2) ; et Paris Sciences et Lettres, dans le cadre de l'Initiative de recherches stratégiques et interdisciplinaires (IRIS) Études globales, qui porte sur l'ensemble des aires. La collaboration entre les UMIFRE et les Comue, encouragée par le CNRS, se met en place, mais d'une manière relativement lente. L'ouverture en 2019 du Campus Condorcet, qui verra une concentration exceptionnelle d'unités de recherche tournées vers les différentes aires mondiales, marquera sans doute une évolution.

Il est indiscutable que l'installation des Comue dans le paysage de l'enseignement supérieur et de la recherche (ESR) français ouvre une période complexe pour le devenir de certaines unités de recherche dans le domaine des études aréales, qui ont à présent pour tutelles des établissements appartenant à des Comue différentes. Cela peut affaiblir des dynamiques de coopération établies de longue date entre les chercheur.e.s, mais peut aussi permettre de nouvelles coopérations transaréales, voire interdisciplinaires, avec d'autres unités de la même Comue. Dans ce domaine, comme dans bien d'autres, beaucoup dépendra de l'engagement des communautés scientifiques.

Une réponse peut être, par ailleurs, un développement encore accru des réseaux de recherche. Les groupements d'intérêt 
scientifique articulent, en effet, une mission d'observation de leurs aires et domaines de compétences avec un rôle de mise en relation des unités de recherche et des chercheur.e.s elles-mêmes et eux-mêmes, à travers les réseaux qu'elles et ils animent et les congrès nationaux qu'elles et ils organisent régulièrement (comme les Rencontres des Études africaines en France ou les Congrès du Réseau Asie).

L'attention des GIS portée aux réseaux de jeunes chercheur.e.s est notable et particulièrement efficace. À titre d'exemple, l'Institut des Amériques attribue chaque année, sur appel à candidatures, quatre contrats doctoraux dont l'originalité est que les étudiants en question sont affectés dans des pôles de l'IDA situés sur le continent américain où ils valorisent la recherche française auprès des partenaires locaux. Ce dispositif devrait être étendu aux autres GIS.

Les GIS, comme, ponctuellement, sur certaines thématiques, des groupements de recherche (GdR), plus souples dans leur fonctionnement, sont donc des outils particulièrement adaptés pour maintenir des liens entre les grands sites de recherche qui sont en train de se mettre en place.

L'IISMM peut avoir une valeur de modèle en ce qui concerne le développement des interfaces entre la recherche et la société. Il dispose de locaux à Paris (boulevard Raspail) et d'une équipe de direction assistée de trois personnels administratifs. Certaines des missions de l'IISMM (comme la diffusion de l'information scientifique) sont communes à celles du GIS 
Moyen-Orient et mondes musulmans alors que d'autres, en particulier dans le domaine de la formation continue, sont propres et connaissent actuellement un essor remarquable. La transformation récente de l'IISMM en UMS commune au CNRS et à l'EHESS, qui servira également d'appui au GIS Moyen-Orient et mondes musulmans, devrait permettre à ses missions de connaître une nouvelle accélération.

L'Institut des Amériques dispose quant à lui, au sein de sa cellule permanente, de chargés de mission qui cherchent à la fois à développer les relations avec le monde économique et à valoriser la recherche française à l'international, leur action étant facilitée par le statut de partenaire privilégié de la Fondation Union européenne-Amérique latine et Caraïbes (EU-LAC) du GIS. 



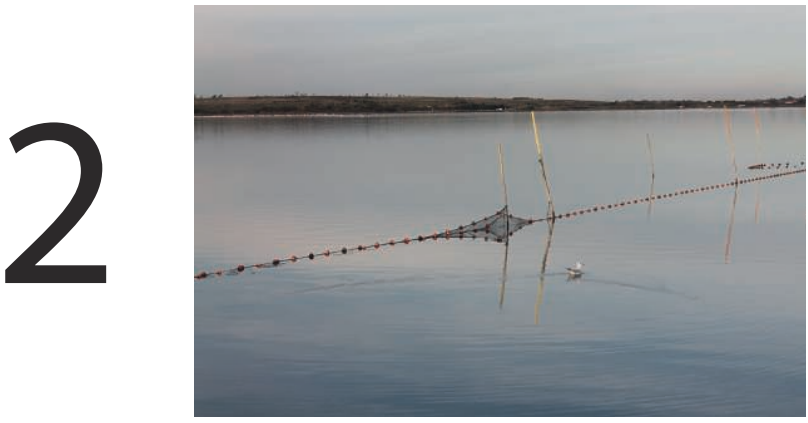

\section{Une question transversale : la qualité de la formation}

La qualité de la formation universitaire initiale est la pierre de touche d'un dispositif efficace en études aréales.

\section{Apprentissage des langues ; apprentissages disciplinaires et pluridisciplinaires}

Même s’il y a des différences importantes entre les pays, ou les terrains dont la langue (ou la langue véhiculaire ou majoritaire) fait partie des langues mondialisées (anglais, espagnol, portugais, français...) et ceux qui réclamentla maîtrise d'une ou plusieurs langues autochtones, la question de l'apprentissage 
des langues dès le début de la formation universitaire est une question clé. Elle se décline de deux manières : la première est celle de l'apprentissage en France ; la seconde est celle de l'apprentissage dans le pays ou sur le terrain étudié grâce à des bourses de mobilité dès les premières années de la formation, actuellement peu nombreuses en France. Les grandes écoles et les universités doivent travailler de manière plus régulière et étroite avec les UMIFRE, par exemple par l'envoi plus systématique d'étudiant.e.s, dès le niveau master.

Un des problèmes récurrents pour les études aréales est l'absence généralisée de cursus intégrés qui regrouperaient la formation à l'aire, la formation à la discipline (histoire, anthropologie, sciences politiques, philosophie...) et la formation à la langue (ou aux langues). Dans certains établissements, les parcours de langue s'efforcent de donner aux étudiant.e.s une solide formation en sciences humaines et sociales avec des unités d'enseignement obligatoires. C'est le cas, par exemple, à Aix-Marseille Université en licence d'arabe, parcours «Langues orientales et sciences humaines et sociales », ou encore à l'Inalco. Le développement des doubles licences dans la plupart des universités pourrait être une première réponse à cette difficulté, comme le montre la double licence histoire-arabe ouverte à Paris-Sorbonne en septembre 2012, qui semble rencontrer un certain succès. Notons, dans le cadre d'une politique volontariste qui est à saluer, l'ouverture en septembre 2016 d'une licence en histoire et civilisations islamiques à l'université de Strasbourg, autour d'une MCF en islamologie. La question de l'enseignement de la langue 
arabe à l'université est cruciale pour la réussite d'une telle formation.

Placé au centre du dispositif licence-master-doctorat (LMD), le master est devenu l'échelon décisif de la formation. C'est le moment où, dans beaucoup de disciplines des sciences humaines et sociales, se rencontrent les flux d'étudiant.e.s formé.e.s à l'université et celles et ceux formé.e.s dans les classes préparatoires aux grandes écoles (CPGE). C'est le moment où se font les choix entre une première forme de professionnalisation (y compris pour les métiers de l'enseignement) ou la poursuite d'un master « recherche ». Le master devrait faire l'objet d'une attention particulière pour que soient proposées aux étudiant.e.s des formations - master ou double master - bien identifiées. C'est également à ce niveau que les étudiant.e.s en études aréales pourraient être initié.e.s, lorsque le besoin scientifique s'en fait sentir, à l'utilisation des systèmes d'information géographique et géolocalisation (SIG), des outils de modélisation et de formalisation, de l'imagerie ou encore des systèmes complexes.

\section{Les études doctorales}

Les études aréales concentrent une proportion non négligeable des thèses soutenues en France tous les ans en sciences humaines et sociales. Rappelons que 14400 doctorats ont été délivrés durant l'année civile 2014 dont 21 \% (environ 3 020) relevaient des sciences humaines ou des humanités, et $14 \%$ 
(environ 2 000) des sciences de la sociétél. Or, les thèses soutenues en SHS sur l'Afrique représentent actuellement à peu près 400 thèses par an et 237 docteur.e.s ont soutenu une thèse sur l'Asie en 2010. À partir de ces éléments, la proportion des thèses ayant une dimension aréale peut être estimée entre un cinquième et un quart des thèses soutenues annuellement en France en SHS.

Le grand nombre de thèses renvoie au problème fondamental de la qualité de l'encadrement doctoral. Comme nous l'avons souligné plus haut, il existe un décalage important entre le nombre de doctorant.e.s, actuel.le.s et potentiel.le.s, surtout venu.e.s del'étranger, et le nombre de professeur.e.s ou directeur. rice.s de recherche aptes à diriger des thèses. Dans plusieurs disciplines ou spécialités, un nombre important de départs à la retraite récents (par exemple, sur le Moyen-Orient et les mondes musulmans, en islamologie, en littérature classique, en archéologie, en études sur le Maghreb, en sciences sociales de l'Iran, et même en histoire du Proche-Orient arabe contemporain) entraîne un véritable déficit en HDR. Cette situation fait qu’un nombre négligeable de thèses sur les aires mondiales est de fait dirigé par des spécialistes de la thématique choisie par la ou le doctorant.e mais pas de l'aire sur laquelle elle ou il travaille. Il y a là une source grandissante de difficultés potentielles.

C'est d'autant plus important que la formation doctorale en France reste trop souvent balbutiante dans les disciplines

1. http://www.enseignementsup-recherche.gouv.fr/cid105632/1-État-emploiscientifique-france-edition-2016.html 
relevant des sciences sociales et surtout des humanités car le travail individuel de recherche l'emporte sur toute autre considération. Or, les doctorant.e.s devraient être accompagné.e.s de manière beaucoup plus intensive en ce qui concerne trois éléments clés : l'écriture scientifique d’abord ; ensuite, la connaissance des systèmes académiques français et européens, qui est trop souvent considérée comme devant être apprise au fil de l'eau, ce qui est la source d'une forme insidieuse d'inégalité ; enfin, les langues, aussi bien les langues de leur terrain que l'anglais qui est actuellement la langue de communication de la science à l'échelle mondiale.

La capacité des doctorant.e.s à écrire et à communiquer en anglais doit être partout améliorée. Cette formation reçue par les doctorant.e.s doit être poursuivie par les postdoctorant.e.s voire les enseignant.e.s-chercheur.e.s et chercheur.e.s titulaires par le biais de stages, d'ateliers ou de summer schools (philologie, paléographie, langues). Mais la capacité des chercheur.e.s en études aréales à publier dans leurs langues de terrain, qui témoigne de leur insertion dans les communautés scientifiques avec lesquelles ils travaillent, doit être aussi valorisée.

Ces particularités linguistiques des spécialistes des études aréales, comme la question abordée plus bas de l'accès aux terrains, ouvrent le dossier du déroulement des thèses dont les règles viennent d'être reprécisées par l'arrêté du 25 mai 2016 fixant le cadre national de la formation et des modalités conduisant à la délivrance du diplôme national du 
doctorat. Les écoles doctorales et les comités de suivi de thèse qui se prononceront en particulier pour le renouvellement des inscriptions au-delà de la troisième année de thèse, s’il est nécessaire, doivent être appelés à tenir compte de la spécificité des spécialistes des études aréales en matière d’apprentissages linguistiques ou de fréquentation des terrains. La reconnaissance des travaux de terrain (et de leurs risques éventuels) pourrait d'ailleurs apparaître dans le contrat de thèse.

Cette réflexion est d'autant plus importante que la connaissance de terrains et de langues fait des spécialistes des études aréales des docteur.e.s qui peuvent trouver à s'employer en dehors du milieu de la recherche, dans les administrations internationales et nationales, dans le monde des organisations non gouvernementales (ONG) et des associations, ou dans le monde de l'entreprise. En lien avec les objectifs ministériels de valorisation du doctorat, en particulier en sciences humaines et sociales, les écoles doctorales devraient tenir compte des spécificités des études aréales.

\section{Le secondaire, un maillon essentiel}

La formation secondaire est un maillon essentiel de la chaîne. En dépit de la difficulté à mener à bien les réformes des programmes scolaires, une première question qui doit être posée est celle de l'enseignement des langues qui alterne succès (le chinois) et difficultés (arabe, hébreu et la plupart des langues orientales). 
Une partie des disciplines fondamentales des études aréales n'est pas enseignée dans le secondaire ; c'est le cas en particulier de l'anthropologie et de la sociologie. Une autre partie de ces disciplines, en particulier l'histoire, la géographie, est enseignée à des niveaux différents du cursus et selon des horaires très différents selonles filières. Or, la situation des aires varie considérablement. Les littératures ou les philosophies non européennes ne sont ainsi que marginalement enseignées. Si l'histoire de l'Asie ou celle des Amériques sont évoquées à différents niveaux du cursus, l'histoire de l'Afrique, en dehors du contexte spécifique de la colonisation ou des relations internationales contemporaines, est étonnamment absente. Dans cette discipline, les questions de concours (CAPES, agrégation) ne font par ailleurs aucune place à l'histoire proprement africaine, et ce depuis des décennies, éloignant encore davantage les futurs enseignant.e.s d'une spécialité qui n'est apparue que quelques années dans les programmes. En géographie, la place de l'Afrique a été récemment réévaluée. Le programme de quatrième fait intervenir, dans le thème consacré aux espaces transformés par la mondialisation, un sous-thème consacré aux dynamiques d’un grand ensemble géographique africain (au choix : Afrique de l'Ouest, Afrique orientale, Afrique australe). En terminale, l'Afrique est à nouveau abordée par le biais de la mondialisation ainsi qu’à travers l'étude du Sahara et de l’Afrique du Sud.

Même si la question de la réforme des programmes scolaires demeure délicate dans notre pays, une discussion pourrait être ouverte entre les groupements d'intérêt scientifique 
et les autorités en charge des programmes du secondaire pour procéder, à tout le moins, à une évaluation globale de la situation. Notons qu'afin de toucher ces publics avec de nouveaux outils, l'Institut des Amériques développe un programme de formats vidéo diffusés en ligne, mettant l'accent sur les nouvelles formes de communication du savoir scientifique. 


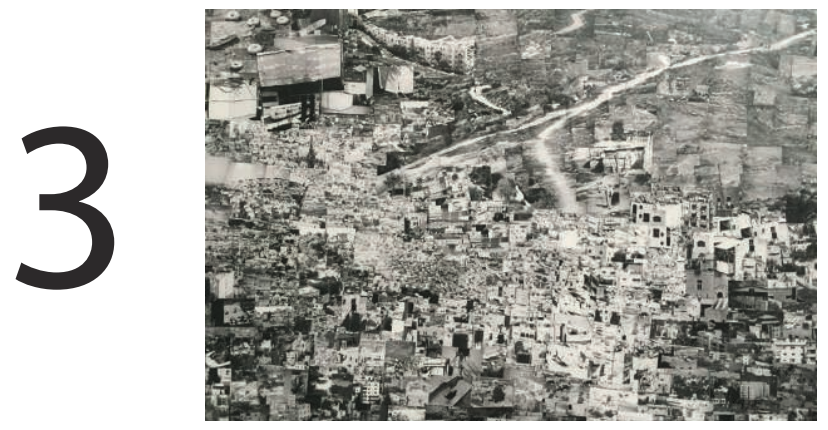

\section{Les spécialistes d'études aréales : des communautés aux besoins spécifiques}

\section{L'accès aux terrains}

Les études aréales, intégrées dans le dispositif global de la recherche en sciences sociales et en humanités qu'elles n'entendent naturellement pas résumer, sont un formidable vecteur de pluridisciplinarité et d'internationalisation de tous les champs de la recherche. Il s'agit d'un secteur où la valeur ajoutée de la recherche française, au sein de la recherche mondiale, est extrêmement élevée. Il convient de développer sans relâche cette dimension, en particulier en s'appuyant sur le dispositif mis en place par l'État pour soutenir la recherche publique hors de France et hors d'Europe : les écoles 
françaises à l'étranger ; les UMIFRE (ministère de l'Europe et des Affaires étrangères, CNRS) ; les programmes d'études à l'étranger subventionnés par le ministère de l'Europe et des Affaires étrangères (partenariats Hubert Curien) ou du CNRS ; les dispositifs mis en place par les établissements.

Le MEAE et le CNRS ont donné aux UMIFRE une triple mission : développer les recherches en sciences humaines et sociales selon des axes scientifiques définis par les tutelles; développer la coopération entre les sciences dans une perspective interdisciplinaire ; enfin, développer les relations entre le monde universitaire français au sens large et la communauté scientifique du pays ou de la région où elles sont implantées.

Les UMIFRE jouent donc un rôle très spécifique par leur capacité à accueillir des chercheur.e.s du CNRS, qui par le biais d'affectations, ou des enseignant.e.s-chercheur.e.s, par le biais de délégations, renouent avec le travail de terrain pendant plusieurs années. À ce propos, il est clair que le dispositif de recherche français doit également s'orienter vers des bourses ou des postdoctorats de longue durée. Les financements d'un an ou moins, surtout lorsqu'ils ne sont pas renouvelables, nepermettent pas un vrai enracinement dans le pays et dans la langue.

Sur place, les UMIFRE doivent pouvoir accueillir aussi une grande diversité de disciplines (y compris linguistiques et littéraires) et pas uniquement en histoire, sociologie, science politique et anthropologie. Il s'agit également de favoriser une politique permettant transversalités et complémentarités 
entre UMIFRE d'une part et entre UMIFRE et écoles françaises à l'étranger d'autre part, à tous les niveaux (politique d'acquisition des bibliothèques, voire politique immobilière commune ; programmes de recherche sur la longue durée sur un objet commun, comme cela commence à se faire, etc.).

La question de l'accès aux terrains ne se limite cependant pas à la capacité de la France à organiser et à soutenir les missions de recherche de ses chercheur.e.s à l'étranger. Elle concerne également l'évaluation des risques que présente une mission de recherche hors du territoire national. Les organismes de recherche comme les établissements d'enseignement supérieur et de recherche respectent les procédures établies à cette fin par les autorités publiques, qui passent généralement par l'avis d'un fonctionnaire défense. L'application de ces règles peut cependant obérer le bon déroulement de projets de recherche, souvent engagés de longue date ou mis en place dans un contexte géopolitique plus stable. Ces projets peuvent être indispensables à une meilleure connaissance de l'aire étudiée, dans une perspective de transfert des connaissances vers la puissance publique. Une réflexion doit donc être engagée sur ce point pour parvenir à un équilibre satisfaisant entre une meilleure intégration en amont de la problématique de la sécurité et, en aval, une concertation maximale des différents acteurs pour arriver à la meilleure évaluation possible des risques.

Dans l'autre sens se pose la question des visas nécessaires aux chercheur.e.s pour travailler dans un pays étranger. Dans la pratique, la plupart des missions de terrain se font avec 
un visa ou une autorisation d'entrée de courte durée, du type «touriste », ce qui fait que la ou le chercheur.e exerce une activité qui n'est pas, sur le fond, couverte par le visa. Pour les séjours plus longs, il faut en passer par un visa de type « scientifique », qui implique un accord avec un partenaire local en général, et qui peut de ce fait considérablement orienter le type de recherche (sans compter le fait que les activités de terrain ne sont pas toujours autorisées dans ce cadre).

\section{L'accès aux données}

Les chercheur.e.s en études aréales peuvent s'appuyer sur un dispositif d'information scientifique et technique (IST) qui joue sur plusieurs niveaux. Le premier est celui de la Bibliothèque nationale de France et des bibliothèques universitaires générales comme la Bibliothèque universitaire des langues et civilisations (Bulac) qui rassemble plus d'un million d'ouvrages concernant toutes les aires de l'Europe balkanique, centrale et orientale à l'Asie et à l'Océanie.

Le deuxième niveau est celui des bibliothèques spécialisées du monde universitaire. Elles sont le plus souvent liées à un centre de recherche, comme par exemple, pour les études africaines, la Bibliothèque du Centre d'études africaines (EHESS), la Bibliothèque de recherches africaines (Institut des mondes africains (IMAF), Paris 1), la bibliothèque Claude-Lévi-Strauss (Laboratoire d'anthropologie sociale (Las), Collège de France) ou encore les bibliothèques Éric-de-Dampierre (Laboratoire d'ethnologie et de sociologie 
comparative (LESC), Paris-Nanterre), du laboratoire « Les Afriques dans le monde » (Bordeaux) ou encore de la Maison méditerranéenne des sciences de l'homme d'Aix-Marseille. Elles peuvent être aussi liées à un centre de formation comme la Bibliothèque d'art et d'archéologie du Centre Michelet (Paris 1 et Paris IV) ou la bibliothèque de géographie (Paris l, Paris IV, Paris 7) qui est une partie de la Bibliothèque interuniversitaire de la Sorbonne (BIS), ou encore la bibliothèque Pierre-Monbeig, sur l’Amérique latine, qui regroupe la plus grande collection en France sur cette aire culturelle.

La troisième strate est celle des bibliothèques hors du monde universitaire : médiathèque du musée du Quai Branly ; Bibliothèque centrale du Muséum national d'histoire naturelle ; bibliothèques de l'IRD et du Centre de coopération internationale en recherche agronomique pour le développement (CIRAD) ; bibliothèques de la FMSH ou encore de l'Académie des sciences d'outre-mer. Notons que, du point de vue des études islamiques en arabe, l'Institut dominicain d'études orientales du Caire est aujourd'hui la bibliothèque française la plus utile grâce à l'extrême cohérence de sa politique d'achat et à son extraordinaire catalogue numérisé.

Ces bibliothèques abritent des fonds de tailles très inégales : elles vont de fonds inférieurs à 10000 livres jusqu'au million de documents abrités par la Bulac. Ces fonds mêlent d'ailleurs souvent livres, périodiques, documents iconographiques, archives voire objets. Une de leurs caractéristiques communes est que leurs collections ont été composées à partir d'achats 
réalisés par la bibliothèque ou par les unités dont elles dépendent, mais aussi à partir de fonds collectés ou déposés par des chercheur.e.s parfois à une date ancienne ou parfois très récemment. Cette pratique - la collecte des archives de chercheur.e.s et leur traitement - qui traverse la question du devenir des archives de la recherche et doit constituer un axe de réflexion, suppose des moyens spécifiques.

Les missions et les centres d'intérêt de ces bibliothèques et dépôts d'archives sont très différents, et cette diversité est un atout à préserver. Ensemble, ils n'en constituent pas moins le socle sur lequel la France peut développer une politique de formation et de recherche sur les études aréales de niveau mondial. L'intégration de la plupart des catalogues de ces bibliothèques, souvent très spécialisées, dans le système universitaire de documentation (Sudoc) révèle d'ailleurs l'ampleur de leurs collections. Ce mouvement d'intégration, essentiel pour la mise à la disposition des fonds à un public étendu, doit être encouragé.

La formation d'une fédération ou d'un conseil de ces bibliothèques, où pourraient être élaborées des stratégies communes, et mises en place des procédures d'harmonisation des politiques d'achat, pourrait permettre de renforcer ce mouvement.

Elle pourrait concerner soit les différentes aires, sur le modèle du réseau national DocAsie, créé en 2007 à Marseille, qui regroupe sur la base du volontariat les documentalistes et bibliothécaires travaillant sur l’Asie, soit les études aréales 
dans leur ensemble. Une telle structure permettrait d'offrir aux publics des bibliothèques une véritable plateforme commune d'accompagnement de la recherche.

Ces bibliothèques rencontrent des difficultés qui leur sont communes. Le premier point concerne les moyens consacrés à l'entrée des ouvrages dans les collections, qui dépendent à la fois des sommes allouées à l'achat matériel des livres et des ressources en personnel liées au traitement de ces acquisitions. Les crédits d'achat ne peuvent être pensés en dehors des affectations des personnels titulaires qui cataloguent et rendent disponibles les livres.

Le deuxième point concerne les compétences spécifiques des personnels des bibliothèques liées aux études aréales. Si l'anglais ou certaines des langues parlées dans les aires culturelles couvertes (espagnol, portugais...) ne posent guère de difficultés, la caractéristique des bibliothèques aréales est de traiter une documentation en langue originale. Le recours au personnel en contrat à durée déterminée (CDD), s'il permet de maintenir le dispositif à flot, empêche la mise en place de politiques d'achat et de catalogage sur la longue durée. Il est donc impératif de créer et financer des postes de bibliothécaires spécialisé.e.s sur les différentes aires, en particulier orientalistes au sens large.

Dans ce cadre, la question de la relation entre la formation offerte par l'École nationale supérieure de l'information et des bibliothèques (ENSSIB) et les besoins des bibliothèques 
aréales doit être repensée : il s’agit d'identifier les compétences, en particulier linguistiques et scientifiques, à valoriser à l'entrée à l'ENSSIB, de manière à s'assurer qu'en sortent des conservateurs formés pour acheter et cataloguer des livres venus aussi bien du Brésil que du Japon ou d’Indonésie.

Au-delà de la question des budgets, la capacité des bibliothèques à être proactives sur les marchés du livre scientifique est essentielle. Une réflexion commune devrait être menée sur les procédures d'acquisition des ouvrages auprès des maisons d'édition internationales non seulement européennes ou nordet sud-américaines mais également implantées dans les pays africains, arabes ou asiatiques. Les échanges des bibliothécaires des grandes bibliothèques d'études aréales avec leurs homologues dans le monde et avec les grandes institutions culturelles doiventêtre encouragés. Àtitre d'exemple, labibliothèque PierreMonbeig a maintenu vivant un fonds de près de 400 revues sur l'Amérique latine grâce à des échanges réciproques de revues locales, dans un modèle intéressant de coopération entre la bibliothèque et la maison d'édition de l'IHEAL.

La question de l'accès aux ressources en ligne des bibliothèques et institutions culturelles des pays étudiés doit être envisagée avec un niveau de granularité suffisamment fin pour que toutes les tailles de ressources numériques ou de banques de données, y compris les plus petites lorsqu'elles sont pertinentes, soient prises en compte. L'ensemble des acteurs, des communautés les plus étendues aux chercheur.e.s individuel.le.s, doivent pouvoir voir leurs besoins d'accès pris en considération. 
La question de l'accès aux ressources documentaires des chercheur.e.s en études aréales inséré.e.s dans des unités de formation et de recherche (UFR) ou des unités de recherche disciplinaires est cruciale.

Une évolution majeure qui se dessine est la naissance du grand équipement documentaire (GED) du Campus Condorcet. La stratégie de recherche du Campus Condorcet a été initialement construite autour de son GED, qui doit recevoir les fonds d'une partie importante des unités parisiennes spécialisées en études aréales, ainsi que les collections de la bibliothèque de la FMSH. Plusieurs défis attendent le GED et son équipe de préfiguration : l'organisation des collections dans ce qui doit être une bibliothèque unifiée ; la création d'une politique d'acquisition en articulation étroite avec les besoin des unités et des chercheur.e.s ; l'articulation entre les collections papier et la documentation numérique.

\section{L'accès aux financements}

L'accès aux terrains d'études dépend essentiellement des financements mobilisés pour la recherche individuelle ou collective. Les organismes et établissements les plus impliqués dans la recherche aréale consacrent une partie importante de leurs financements aux dotations des unités dans ce domaine. Celles des UMR en études aréales sont, par exemple, parmi les plus élevées accordées chaque année par l'Institut des sciences humaines et sociales du CNRS. Mais les dotations des unités agissent aussi de façon efficace à l'échelle individuelle ou à celle 
des programmes de petite taille. La place des études aréales dans les dispositifs de financement sur projet est donc centrale.

Les financements pour les missions de courte ou moyenne durée sont déjà bien développés, aussi bien dans les ministères (comme les partenariats Hubert Curien du ministère de l'Europe et des Affaires étrangères) que dans les organismes (comme les soutiens à la mobilité internationale du CNRS), ou dans les établissements, par le biais des Labex ou des programmes labellisés par les initiatives d'excellence (IDEX) par exemple. Bien des pays étrangers, comme le Japon ou le Brésil, ont par ailleurs mis en place d'efficaces programmes d'aide pour les doctorant.e.s et chercheur.e.s étranger.ère.s. Outre le fait que ces financements visent parfois davantage une coopération entre universités que le soutien à des recherches de terrain, il ne convient de toute façon pas que la recherche nationale soit, pour certains terrains, trop dépendante des programmes locaux, qui ont leurs propres spécificités et orientations. Enfin, la montée en régime de la recherche nationale au sein des nouvelles puissances mondiales ou des pays émergents diminue progressivement chez eux le besoin de recourir aux spécialistes occidentaux.

Pour les projets de grande ampleur, les programmes de l'Agence nationale de la recherche (ANR) ont un rôle spécifique à jouer. Pour prendre un seul exemple, les ANR Trans-TUR et Trans-FAIRE ont permis de structurer une vraie communauté de chercheur.e.s sur la Turquie, animée par une dynamique collective et interdisciplinaire portée 
par une jeune génération et renforcée par l’attractivité de la Turquie et la qualité scientifique des chercheur.e.s turc.que.s, malgré la situation difficile dans laquelle elles et ils se trouvent actuellement. Nous pourrions citer également « GlobAfrica, Reconnecter l'Afrique », porté par l'Institut français d'Afrique du Sud (IFAS) - USR 3336

L'ANR doit donc s'assurer que les projets liés aux études aréales puissent être évalués dans les meilleures conditions aussi bien dans ses appels à projet génériques que dans les nouveaux programmes que sont l'instrument Jeunes chercheuses/Jeunes chercheurs et le programme baptisé Tremplin-ERC (T-ERC). L'ANR pourrait être également encouragée à mettre en place des actions spécifiques sur les études aréales dans le cadre des collaborations qu'elle développe avec les agences de financement de la recherche d'autres pays.

Enfin, ces communautés scientifiques doivent être incitées à penser dans le cadre européen. Cela passe par l'encouragement des candidatures de chercheur.e.s de ces champs aux European Institutes for Advanced Studies ainsi que par les demandes d'European Research Council (ERC). Le CNRS, comme la plupart des Comue, et certains établissements ont d'ailleurs mis en place des dispositifs en ce sens que les spécialistes des études aréales devraient systématiquement utiliser. À titre d'exemple, le GIS IDA a lancé en 2016 un appel à projets pour l'européanisation de ses membres, par lequel il financera deux projets à hauteur de 12000 euros pour le montage d'un 
réseau de coopération visant, à terme, le dépôt d'un projet H20201. Ce type d'action, au plus près des chercheur.e.s, pourrait être étendu à tous les GIS.

1. Horizon 2020, programme 2014-2020 pour la recherche et l'innovation de l'Union européenne. 

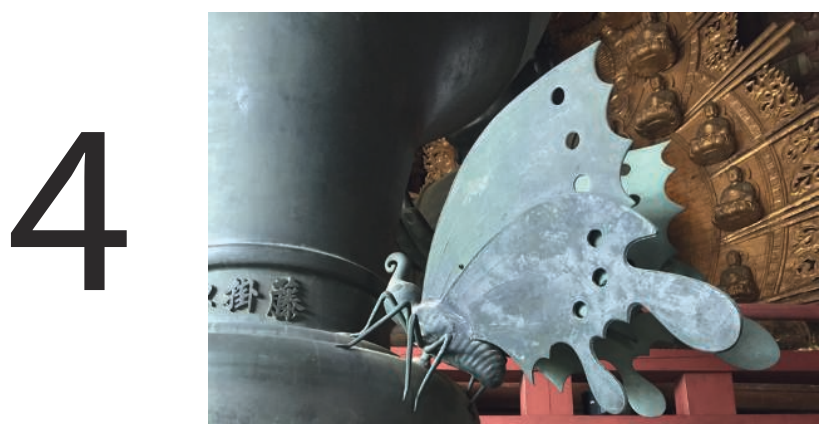

\section{Les recrutements}

La France est un des rares pays européens qui conserve un dispositif efficace de recrutement de chercheur.e.s et d'enseignant.e.s-chercheur.e.s sur un statut de fonctionnaire. Il s'agit d'un atout considérable. Les évolutions récentes du système académique français ont confié aux établissements la maîtrise de leur recrutement, ce qui rend les postes académiques particulièrement sensibles aux décisions prises dans chacun d'entre eux dans le contexte qui leur est propre. Cette situation peut engendrer des risques pour les études aréales comme pour les disciplines rares telles qu'elles ont été définies dans le rapport remis le 16 décembre 2014 au secrétariat d'État à l'Enseignement supérieur et à la Recherche. 


\section{Une stratégie nationale de recrutement pour les études aréales}

Une première réponse passe par la préservation du potentiel d'enseignement et de recherche sur les différentes aires et disciplines concernées. Elle ne peut passer que par une augmentation du nombre de postes tournés vers les études aréales, y compris par le biais de créations, comme cela a pu être fait en 2016 sur les questions liées à l'islam, et par la mise en place de dispositifs visant à harmoniser, à l'échelle nationale, les décisions prises au cas par cas pour le renouvellement des postes existants.

Eu égard à l'enjeu national de la recherche aréale, l'effort ne peut être concentré sur une poignée d'organismes (CNRS, IRD...) ou d'établissements (en particulier EHESS, EPHE, Inalco, Paris 1, Paris 7 ou AMU...), dont l'engagement doit être salué. Il doit, au contraire, être réparti sur l'ensemble du territoire national dans le cadre d'une stratégie concertée. Il est également essentiel que ne se creuse pas un écart entre, d'un côté, les dispositifs des organismes, comme le CNRS, ou les grandes écoles, et de l'autre, les universités.

La réflexion doit porter non seulement sur le maintien ou la création de postes de jeunes chercheur.e.s (chargé.e.s de recherche et maîtres de conférences), mais aussi sur les postes de directeur. rice.s de recherche, directeur.rice.s d'études et professeur.e.s, afin que la capacité d'encadrement, dont nous avons montré la nature fragile dans certains secteurs, soit préservée. 
Le rôle des personnels ingénieurs, techniciens et administratifs (ITA) et BIATSS est particulièrement important dans les unités d'études aréales. Les ingénieur.e.s de recherche, les documentalistes, qui traitent un matériel dans des langues non européennes, les gestionnaires, en charge du suivi de missions lointaines ou accueillant des collègues étrangers, les cartographes ou encoreles secrétaires de rédaction de revues qui jonglent avec des articles écrits en des langues différentes et venant de communautés scientifiques très diverses, apportent leurs compétences propres ainsi qu'une expérience souvent irremplaçable de contacts internationaux. Les efforts de recrutement et d'affectation d'ITA, mais aussi de BIATSS, doivent donc être poursuivis et intensifiés.

Nous devons donc faire avancer, d'une manière globale, l'idée que des postes nouveaux puissent être orientés vers ces secteurs, où les chaires universitaires, les postes de chercheur.e.s et les projets de recherche ont beaucoup à apporter aux étudiant.e.s et à la société, surtout dans le double contexte de globalisation accélérée depuis les années 2000, et de montée actuelle des interrogations sur la sécurité humaine.

\section{La lisibilité des recrutements}

Une seconde réponse passe par une meilleure préparation en amont du vivier des candidat.e.s potentiel.lle.s. Elle se décline à plusieurs niveaux.

Le dispositif de financement des doctorant.e.s et des chercheur.e.s en études aréales doit être ainsi rendu plus lisible 
par une centralisation de l'information et de sa diffusion. L’impulsion donnée par les dispositifs nés du programme d'investissements d'avenir (PIA), en particulier les Labex, pour implanter dans les SHS françaises le contrat postdoctoral a été essentielle, mais elle s'est accompagnée d'une dispersion des propositions, voire d'une relative opacité de leur distribution, qui contraste avec les normes internationales.

De même, la place des contrats postdoctoraux dans la carrière des chercheur.e.s et des enseignant.e.s-chercheur.e.s doit être repensée, dans une démarche qui dépasse d'ailleurs les études aréales. Le contrat postdoctoral est actuellement conçu comme une solution d'attente plus que comme une étape en soi de la carrière académique permettant une recherche originale sur le terrain. Le développement de postdoctorats de longue durée (trois ans) et à un haut niveau de rémunération permettrait une évolution de cette situation. L'ouverture aux chercheur.e.s étranger.ère.s des postdoctorats accordés par les établissements français devrait être une priorité, surtout pour ceux qui ont un meilleur niveau en langue ou dans la discipline initiale.

La question de la visibilité des études aréales est cruciale et un système de prix, médailles et récompenses doit être pensé dans ce cadre précis. Nous proposons de créer pour chacune des aires, ou au sein de tous les GIS d'études aréales évoqués ici, une hiérarchie de prix multidisciplinaires distinguant les chercheur.e.s à différents stades de leur parcours académique. Les prix mis en place par l'IISMM et le GIS Moyen-Orient et mondes musulmans, ou encore le prix de thèse du GIS Institut 
des Amériques, qui permet de publier la thèse récompensée dans une des collections de l'IDA, sont de bons modèles de ce qui pourrait être systématisé.

Enfin, une réflexion spécifique doit être engagée sur les disciplines « à agrégation », en particulier là où l'agrégation, concours de recrutement de professeurs du secondaire, sert de facto de critère de recrutement des contrats doctoraux par les écoles doctorales, voire de facteur discriminant dans le recrutement des maîtres de conférences.

\section{Les encouragements à la mobilité entrante et sortante}

La qualité des recrutements faits par les organismes, les écoles et les universités en France sera d’autant plus élevée que les candidats, à tous les niveaux de recrutement, y compris au niveau directeur.rice de recherche (DR), directeur.rice d'étude (DE) ou PU, seront choisis dans un vivier international.

Les recrutements de collègues étranger.ère.s seront facilités par l'intensification de la mobilité entrante de chercheur.e.s et enseignant.e.s-chercheur.e.s, y compris originaires des pays du Sud. La politique de visas menée par la France en direction des universitaires étranger.ère.s doit donc être menée dans l'objectif de faciliter la venue de ces collègues ainsi que des étudiant.e.s. La mise en place d'une réflexion commune avec le ministère de l'Europe et des Affaires étrangères est indispensable sur ce point. 
Pour fluidifier ces échanges, la France doit développer l'accueil des grands congrès internationaux d'études aréales sur le modèle ouvert par le GIS Études africaines en France qui a organisé en 2015 le congrès de l'association européenne African-European Group for Interdisciplinary Studies, en 2015. Le centre de colloques du Campus Condorcet pourra être une plateforme particulièrement utile en ce sens. L'Institut des Amériques prévoit d'y organiser en 2019 un grand congrès des américanistes. 


\section{Conclusion}

Dans un climat d'intense concurrence scientifique, la mise en place des recommandations présentées doit permettre à la science française de retrouver une place de leader dans l'analyse des sociétés non occidentales, pour faire entendre une voix méthodologique et analytique spécifique. Les groupements d'intérêt scientifique, créés pour trois d'entre eux entre 2012 et 2013, arrivent au terme de leur premier mandat. Ils ont fait la preuve de leur efficacité mais un nouveau temps s'ouvre devant eux, pour lequel ils doivent redéfinir leurs missions et, avec l'aide des communautés scientifiques aussi bien que des pouvoirs publics, intensifier leurs actions. Les GIS souhaitent, en particulier, que les ministères concernés et les établissements entendent les recommandations faites par les communautés scientifiques, a fortiori en matière de recrutement. L'ensemble des acteurs de l'ESR doivent œuvrer dans le même sens à partir d'une vision ouverte et globale pour que le système gagne en efficacité et en énergie. 



\section{Table des matières}

Sommaire

Introduction

Préconisations

21

1. Un développement plus intégré des formations $\quad 22$

2. Un soutien ciblé à la recherche $\quad 24$

3. Une stratégie nationale de recrutement pour les études aréales

4. Le rôle des nouvelles institutions : vers une convergence des efforts?

L'état de la recherche : un constat différencié selon les aires et selon les disciplines.

1. Analyse générale

2. Un regard contrasté sur les effectifs

3. Études aréales et politique de site

Une question transversale :

la qualité de la formation 
1. Apprentissage des langues; apprentissages

disciplinaires et pluridisciplinaires

2. Les études doctorales $\quad 47$

3. Le secondaire, un maillon essentiel $\quad 50$

Les spécialistes d'études aréales:

des communautés aux besoins spécifiques _ $\quad 53$

1. L'accès aux terrains

2. L'accès aux données $\quad 56$

3. L'accès aux financements ﹎..................................... 61

Les recrutements $\quad 65$

1. Une stratégie nationale de recrutement pour les études aréales $\quad 66$

2. La lisibilité des recrutements 67

3. Les encouragements à la mobilité entrante et sortante $\quad 69$

Conclusion 71

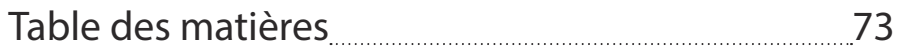

Table des sigles _ $\quad 75$

Table des illustrations $\quad 81$ 


\section{Table des sigles}

\section{AMU}

Aix-Marseille Université

ANR

Agence nationale de la recherche

\section{Athéna}

Alliance thématique nationale des sciences humaines et sociales

\section{BAP}

branche d'activité professionnelle

\section{BIATSS}

bibliothèques, ingénieurs, administratifs, techniques, sociaux et santé

\section{BIS}

Bibliothèque interuniversitaire de la Sorbonne

\section{Bulac}

Bibliothèque universitaire des langues et civilisations

\section{CAPES}

certificat d'aptitude au professorat de l'enseignement du second degré CDD

contrat à durée déterminée

\section{CIRAD}

Centre de coopération internationale en recherche agronomique pour le développement

\section{CNRS}

Centre national de la recherche scientifique

\section{CoNRS}

Comité national de la recherche scientifique

\section{CNU}

Conseil national des universités 


\section{Comue}

communauté d'universités et d'établissements

CPGE

classes préparatoires aux grandes écoles

CPU

Conférence des présidents d'université

CRCT

congés pour recherches et conversions thématiques

DE

directeur.rice d'étude

DR

directeur.rice de recherche

EA

équipe d'accueil

EFEO

École française d'Extrême-Orient

EHESS

École des hautes études en sciences sociales

ENSSIB

École nationale supérieure de l'information et des bibliothèques

EPHE

École pratique des hautes études

ERC

European Research Council

ESR

enseignement supérieur et recherche

EU-LAC

Fondation Union européenne-Amérique latine et Caraïbes

FMSH

Fondation Maison des sciences de l'homme

GDR

groupement de recherche

GED

grand équipement documentaire

GIS

groupement d'intérêt scientifique

HDR

habilitation à diriger des recherches 


\section{HESAM}

Hautes Écoles Sorbonne Arts et Métiers

IDA

Institut des Amériques

IEA

Institut d'études avancées

IDEX

initiatives d'excellence

IFAS

Institut français d'Afrique du Sud

IEP

Institut d'études politiques

IFRI

Institut français de recherche en Iran

IHEAL

Institut des hautes études de l'Amérique latine

IISMM

Institut d'études de l'Islam et des sociétés du monde musulman

IMAF

Institut des mondes africains

Inalco

Institut national des langues et civilisations orientales

INED

Institut national d'études démographiques

IRD

Institut de recherche pour le développement

\section{IREMAM}

Institut de recherches et d'études sur les mondes arabes et musulmans

IRIS

Initiative de recherches stratégiques et interdisciplinaires

\section{ISITE}

Initiatives-Science-Innovation-Territoires-Économie

IST

information scientifique et technique

ITA

ingénieurs, techniciens et administratifs

IUF

Institut universitaire de France 


\section{Labex}

laboratoire d'excellence

\section{LAS}

Laboratoire d'anthropologie sociale

\section{LESC}

Laboratoire d'ethnologie et de sociologie comparative

\section{LMD}

licence-master-doctorat

\section{MEAE}

ministère de l'Europe et des Affaires étrangères

\section{MCF}

maître de conférences

\section{MESRI}

ministère de l'Enseignement supérieur, de la Recherche et de l'Innovation

\section{MMSH}

Maison méditerranéenne des sciences de l'homme

\section{MOM}

Maison de l'Orient et de la Méditerranée

\section{ONG}

organisation non gouvernementale

\section{PIA}

Programme d'investissements d'avenir

\section{PIA 2}

deuxième programme d'investissements d'avenir

PU

professeur.e d'université

\section{SIG}

systèmes d'information géographique et géolocalisation

\section{SHS}

sciences humaines et sociales

\section{Sudoc}

Système universitaire de documentation

\section{UFR}

unités de formation et de recherche

\section{UMIFRE}

unité mixte des instituts français de recherche à l'étranger

\section{UMS}

unité mixte de services 


\section{UMR}

unité mixte de recherche

UPS

unité propre de services au CNRS 



\section{Table des illustrations}

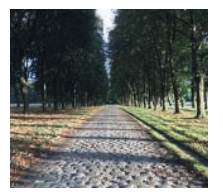

L'état de la recherche : un constat différencié selon les aires et selon les disciplines

"Zébrures» (O) Madeleine B., 2016

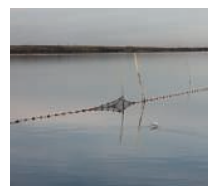

Une question transversale : la qualité de la formation

«Tchekhov» () Madeleine B., 2016

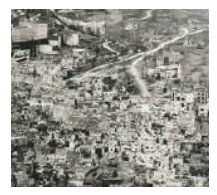

Les spécialistes d'études aréales :

des communautés aux besoins spécifiques «Photocartes» $\odot$ Boise, 2018

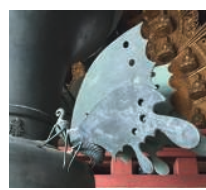

Les recrutements

«Repérages» () Boise, 2018 


Achevé d'imprimer en juillet 2018

sur les presses de l'Imprimerie de l'Ouest parisien

Boulogne-Billancourt

Dépôt légal : juillet 2018 